\title{
Kinematic and dynamic modeling and approximate analysis of a roller chain drive
}

\author{
Fuglede, Niels; Thomsen, Jon Juel
}

Published in:

Journal of Sound and Vibration

Link to article, DOI:

10.1016/j.jsv.2015.12.028

Publication date:

2016

Document Version

Peer reviewed version

Link back to DTU Orbit

Citation (APA):

Fuglede, N., \& Thomsen, J. J. (2016). Kinematic and dynamic modeling and approximate analysis of a roller chain drive. Journal of Sound and Vibration, 366, 447-470. https://doi.org/10.1016/j.jsv.2015.12.028

\section{General rights}

Copyright and moral rights for the publications made accessible in the public portal are retained by the authors and/or other copyright owners and it is a condition of accessing publications that users recognise and abide by the legal requirements associated with these rights.

- Users may download and print one copy of any publication from the public portal for the purpose of private study or research.

- You may not further distribute the material or use it for any profit-making activity or commercial gain

- You may freely distribute the URL identifying the publication in the public portal

If you believe that this document breaches copyright please contact us providing details, and we will remove access to the work immediately and investigate your claim 


\title{
Kinematic and dynamic modeling and approximate
} analysis of a roller chain drive

\author{
Niels Fuglede, Jon Juel Thomsen ${ }^{1}$, \\ Department of Mechanical Engineering, Technical University of Denmark, Building 404, \\ DK-2800, Lyngby, Denmark
}

\begin{abstract}
A simple roller chain drive consisting of two sprockets connected by tight chain spans is investigated. First, a kinematic model is presented which include both spans and sprockets. An approach for calculating the chain wrapping length is presented, which also allows for the exact calculation of sprocket center positions for a given chain length. The kinematic analysis demonstrate that the total length of the chain wrapped around the sprockets generally varies during one tooth period. Analytical predictions for the wrapping length are compared to multibody simulation results and shows very good agreement. It is thereby demonstrated that chain drives with tight chain spans must include compliant components to function. Second, a dynamic model is presented which includes the two spans and the driven sprocket. Assuming the presence of a stationary operating state, the presented dynamic model allows for analytical studies of the coupled motion of the chain spans and driven sprocket. Parametric excitation of the spans come from sprocket angular displacements, and the driven sprocket acts as a boundary which can be compliant in the axial direction. External transverse
\end{abstract}

\footnotetext{
${ }^{1}$ Corresponding author: jjt@mek.dtu.dk
} 
excitation of the spans comes from polygonal action, and is treated through kinematic forcing at the moving string boundaries. Perturbation analysis of the model is carried out using the method of multiple scales. Results show a multitude of internal and external resonance conditions, and some examples are presented of both decoupled and coupled motion. Together, the kinematic and dynamic model are aimed toward providing a framework for conducting and understanding both numerical, and experimental investigations of roller chain drive dynamics.

Keywords: Roller chain drive, axially moving string, moving boundaries, polygonal action, kinematic analysis, multibody simulation

\section{Introduction}

Roller chain drives are applied for power transmission in many mechanical systems due to a high energy efficiency, large power capacities, timing capabilities, flexibility in choosing shaft center distance, and ease of installation and maintenance. However, roller chain drives are also challenging due to the presence of undesired noise and vibration, and is therefore subject to ongoing studies [1].

Kinematic studies of roller chain drives are carried out by modeling the sprockets as polygons [2]. The angular motion of two sprockets connected by a chain span is considered to happen through a series of four-bar mechanisms [3]. Because a chain wrapped around a sprocket forms a polygon rather than a circle, several less desirable effects are introduced. These are referred to as polygonal action and include; impact when a roller seats on a sprocket, non-constant torque transmission and velocity ratio between sprockets. The monograph [4] introduces subjects such as tooth geometry, load distribution, friction, wear, and driven sprocket velocity variations. Studies demonstrate 
how the center distance can be chosen to minimize velocity variations of the driven sprocket [5]. A procedure for calculating the driven sprocket motion for a general chain drive was developed, and examples demonstrated how design parameters influence the polygonal action [6]. An exact and approximate kinematic analysis of the chain drive modeled as a four-bar mechanism was given [7]. The presentation included the derivation of the driven sprocket angular- position, velocity, and acceleration. Seating and release configurations were also determined, and the analytical results were illustrated using three chain drive configurations.

It is common to the above kinematic studies that the analysis only considers the tight span transferring the torque between two sprockets. In this work, the slack span is also considered, which is relevant for practical roller chain drives where the sprockets are positioned to keep all the chain spans in tension during operation, as in e.g. low speed marine propulsion engines. The kinematic analysis of a roller chain drive presented here is for two sprockets with arbitrary number of teeth and center distance. Our results demonstrate that when sprockets are modeled as polygons, and the chain spans are pretensioned to form straight lines, the total wrapping length of the chain varies periodically with the tooth frequency. This leads to a prediction of a tooth-periodic variation of the axial tension in the chain spans. The analytical kinematic prediction is compared to multibody simulation results and shows very good agreement. Simulation results are obtained using a simulation program which include dynamic effects, flexible contact, and the exact geometry of the sprocket teeth $[8,9]$. These results demonstrate that a real roller chain drive must include compliant components for a tensioned chain to wrap around rotating sprockets, as the sprockets are effectively eccentric. The kinematic results allow for the exact prediction of 
the shaft center distance, for which a chain with a given number of links wraps tightly around two sprockets.

Under the assumption that the chain span motion decouples from the dynamics of sprockets and attached machinery, the studies of roller chain drive dynamics is found to border the analysis of axially moving strings, which belongs to the class of gyroscopic systems. For a decoupled axially moving string, equations governing non-linear oscillations were derived using Hamiltons principle, and the influence of transport velocity and nonlinearity on the natural frequencies was analyzed [10], along with examples of the modal distributions [11]. Based on the modal analysis of discrete gyroscopic systems $[12,13]$, the result of exact modal analysis of axially moving continua was presented [14], as well as asymptotic studies of the forced and free response around super- and sub-critical axial velocity [15]. From these studies, the characteristics of axially moving strings are shown to be transport-velocity dependent natural frequencies, the presence of a critical speed, and complex-valued traveling string eigenfunctions utilized by casting the system in a first order form. The complex eigenfunctions are shown to be a superior orthogonal discretization basis, compared to real-valued stationary string eigenfunctions, when predicting parametric instability [16]. For an extensive review of the research on axially moving materials until 2004 we refer to [17].

In this study we analyze the effect of the span being supported by moving boundaries with prescribed endpoint displacements, and demonstrate that a span with a first order variable length introduces a second order effect.

Central to many studies of string dynamics is the presence of parametric excitation from prescribed tension fluctuations, which may lead to parametric instability. Most often these excitations are single frequency har- 
monic excitations, but the response have also been analyzed for multiple frequency excitations [18]. Tension variation can have many sources, e.g. camshaft excitation, eccentricity of belt pulleys or variable transport velocity, cf. the review [19]. The study of sprocket and chain span motion introduced in [20,21] and subsequent studies [22] are based on the assumption that the driven sprocket angular velocity varies time-harmonically at the tooth frequency, due to the excitation coming from the variable velocity ratio between driver and driven sprocket. Comprehensive models are derived for belt drives [23] and engine timing chain drives [24], with non-linear transverse and longitudinal motion of the chain spans coupled to the displacements of pulleys/sprockets with flexible supports. In the case where chain span decouples from the motion of the driven sprocket, the chain span stability was analyzed [24].

Here we present a model which takes into account the coupled motion of span and sprocket angular displacements, i.e. motion of the chain span when it is connected to a sprocket which is compliant, and the sprocket motion is not assumed to be given. We demonstrate how the coupled nonlinear system of the spans and driven sprocket can be analyzed analytically using perturbation methods, and predictions are provided for the amplitude frequency response.

Meshing between the chain span and sprockets introduce problems with noise and wear in real chain drives. It is therefore of interest to study the chain span subjected to transverse excitation at, or near, the span boundaries. One approach is to analyze the impulse created when a roller seats on a sprocket, and assume the impulse to be independent of the chain span dynamic response [25, 26]. Noise and vibration problems has also motivated several experimental studies $[27,28]$. Including the chain vibrations 
in the estimate of the impulse function improves the analytical predictions, and gives better agreement with experimental results $[29,30]$. Predictions of meshing noise that compare well with measurements has also been presented $[31,32]$.

For this study we consider an impulsive excitation acting at the tooth frequency at both span ends, in order to model the kinematic transverse excitation coming from polygonal action. The impulses are assumed to be independent of the span dynamics, and are derived only from the chain geometry and operating speed. The time between the impulses acting at either span end can be determined from the kinematic configuration of the chain drive [7]. This type of excitation is of specific interest to designers of chain drives operating in low-speed marine engines. Our analysis demonstrate how the impulse loading leads to multifrequency excitation, and identifies the operating conditions where the transverse excitation from meshing can be resonant to other excitation sources.

The structure of the paper is as follows: First we present a kinematic model, carry out the analysis for the total wrapping length, and demonstrate the analytical results by some examples. Analytical predictions for the kinematic analysis are then compared to multibody simulation results. Secondly, we derive the governing equations for the coupled chain-sprocket dynamic model, perform an approximate analysis, and present three examples to illustrate the type of results that can be achieved.

\section{Kinematic modeling and analysis}

First we introduce the model assumptions followed by the definition of the coordinate systems and main kinematic parameters. When defining the 
kinematic model we also introduce the seating and release configurations for both spans, as well as the driven sprocket angular position determined as a function of the driver angular position. These kinematic relations provide the initial steps required for the determination of the lower span length, and are based on the analysis [7]. With the seating, release and driven sprocket motion determined, a procedure for determining the lower span length is given. With the lower span length determined, the (approximate) total wrapping length can be calculated, which is the main object of the kinematic analysis.

\subsection{Kinematic model assumptions}

The kinematic model neglects all dynamic effects. It is assumed that the chain spans are tensioned into straight lines, sprockets are rigid and match the chain such that the sprocket pitch is equal to the chain pitch, and rollers are seated at the center of the seating curves. There are no mechanical clearances, and the system is frictionless. The kinematic model is shown in Figure 1.

By modeling the two sprockets and the upper span $a$ as a four-bar mechanism, the length $l_{b}$ of the lower span $b$ is determined as a function of the driver angle $\theta$. As will be shown, this length is not constant, but varies during each tooth period. The length $l_{b}$ is calculated by assuming that span $b$ is the only member which can deform, i.e. for the upper span $a$, as well as the parts of the chain seated on the sprockets, the distance between the rollers equals the chain pitch. It is necessary to make this assumption in order to obtain results for the total wrapping length of a chain using the existing methods, i.e. the chain drive modeled as a four-bar mechanism. In a real chain drive, the deformation would be distributed on all the chain 
drive components, but taking this into account would require a different approach, which is not readily apparent.

The method is exact when the total wrapping length equals an integer number of chain links, since in this case, the chain is not deformed. The analysis thereby provides a method for calculating the exact sprocket center positions for a given chain length. This is of practical relevance, since real chains has a high axial stiffness, and the axial span tension is therefore sensitive to the sprocket center positions. With this analysis we identify and approximately quantify the property of roller chain drives having a variable wrapping length. This effect has been observed for real chains, but has not previously been treated theoretically.

\subsection{Kinematic model}

Figure 1 shows the kinematic model of a chain drive consisting of a driven sprocket $O$ connected by the chain to make both sprockets rotate in the clockwise direction, as prescribed by the driver sprocket $C$. The sprockets are positioned such that the two spans are both in tension and form straight lines. The angular position of the sprockets to the rollers constituting the current span endpoint are given by $\theta_{a}$ and $\phi_{a}$, for the the driver and driven sprockets, respectively; similarly for the lower span, where the span endpoint positions are $\theta_{b}$ and $\phi_{b}$. Subscripts $o, c$ and $a, b$ are used throughout the kinematic analysis to relate variables specific to sprockets $O, C$ and spans $a, b$ respectively, e.g. $\alpha_{o}$ and $\theta_{b}$.

As the sprockets rotate, chain links will recurrently be released from a sprocket to enter the connecting span and seated on a sprocket, thereby being removed from the connecting span; these seat and release configurations are referred to with subscripts $r$ and $s$, respectively. 


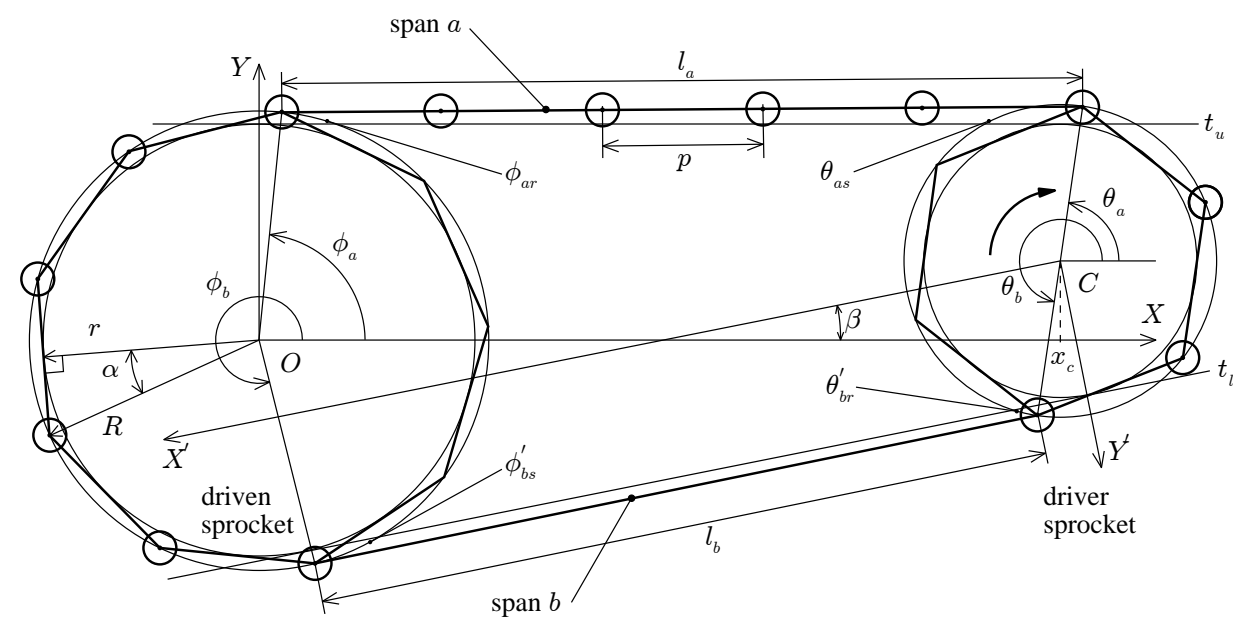

Figure 1: Kinematic model of a chain drive consisting of two sprockets connected by tight spans $a$ and $b$ of instantaneous length $l_{a}$ and $l_{b}$, respectively. The driver sprocket $\mathrm{C}$ rotates counter-clockwise.

A sprocket with $n$ teeth is modeled as a pitch polygon with $n$ sides of length $p$, where $p$ is the chain pitch length. A chain roller seated on a sprocket is positioned such that the center of the sprocket seating curve and the roller center traces out the pitch circle with radius $R=p /(2 \sin \alpha)$, circumscribing the pitch polygon. The circle inscribed of the pitch polygon has radius $r=p /(2 \tan \alpha)$ and the pitch angle is given by $\alpha=\pi / n$, making the angle between two seated rollers $2 \alpha$.

The origin of the inertial $X, Y$ coordinate system coincides with the center of sprocket $O$. It is orientated such that the $X$-axis is parallel with the upper tangent $t_{u}$ which is common to the two inscribed circles. The center position of sprocket $C$ is $\left(x_{c}, y_{c}\right)$, where

$$
x_{c}=(N+1+f) p, \quad y_{c}=r_{o}-r_{c} .
$$

By this definition of $x_{c}$, two parameters have been introduced: The number 
of chain links $N \in \mathbb{N}$ and the pitch fraction $f \in[0,1[$. For the lower span, the $X^{\prime}, Y^{\prime}$ coordinate system has its origin coinciding with the center of sprocket $C$, and is orientated such that the $X^{\prime}$-axis is parallel to the lower tangent $t_{l}$.

For real chain drives, a chain wrapped around two sprockets must contain an inter number of chain pitches. Therefore the pitch fraction $f$ will not be a parameter than can be chosen independently when the spans form straight lines, since the shaft center distance $O C$ must be adjusted to obtain the desired pretension of the chain.

It is necessary to determine the seating and release configurations, as these are required for calculating the chain wrapping length. Seating and release configurations for the upper and lower span can be determined according to [7]: By considering the two sprockets to be connected by one span only, the seating and release configurations are determined from the kinematic analysis of the resulting four-bar mechanism. Following [7], the upper span seating and release configurations are: $\left(\theta_{a s}, \phi_{a s}\right)$ and $\left(\theta_{a r}, \phi_{a r}\right)$, and similarly for the lower span, $\left(\theta_{b s}^{\prime}, \phi_{b s}^{\prime}\right)$ and $\left(\theta_{b r}^{\prime}, \phi_{b r}^{\prime}\right)$. These seating and release configurations are determined in the $X, Y$ and $X^{\prime}, Y^{\prime}$ coordinate systems, respectively.

It is also necessary to determine the driven sprocket angular position and the span length $l_{a}$ as a function of the driver angular position, as it is required for calculating the chain wrapping length. The kinematic motion of the fourbar mechanism with span $a$ can determined according to [7]: By considering the two sprockets to be connected by span $a$ only, the angular position of the driven sprocket can be determined as a function of the angular position of the driver sprocket, i.e. $\phi=\phi(\theta)$. Also, the span length $l_{a}=l_{a}(\theta)$ can be determined according to [7]. The span length $l_{a}$ varies discontinuously with 
the driver angular position, because links enter and leave the span. Here, and in the following, a variable marked with prime denotes angles given in the $X^{\prime}, Y^{\prime}$ coordinate system. In the following, a procedure for determining the lower span length $l_{b}$ will be given.

\subsection{Calculating the lower span length}

It is convenient to consider one period of the angular motion to start from $\theta_{a s}$, the configuration where a roller from the span $a$ seats on the driver sprocket. The upper span length varies according to

$$
l_{a}=(N+1+h) p, \quad h=\left\{\begin{array}{l}
0 \text { for } \theta_{a s} \geq \theta_{a} \geq \theta_{a r}, \\
1 \text { for } \theta_{a r} \geq \theta_{a} \geq \theta_{a s}+2 \alpha_{c} .
\end{array}\right.
$$

Note that $\theta$ and $\phi$ decrease as the sprockets rotates in clockwise direction.

The angle between the two coordinate systems is $\pi+\beta$, where $\beta$ is given by

$$
\beta=2 \arccos \left(x_{c} /|O C|\right) .
$$

The seating and release configurations provides limits for the angles to the span endpoints. Expressing these limits in the $X, Y$ coordinate system gives

$$
\begin{aligned}
\theta_{a} & \in\left[\theta_{a s}-2 \alpha_{c}, \theta_{a s}\right], \\
\phi_{a} & \in\left[\phi_{a r}, \phi_{a r}+2 \alpha_{o}\right], \\
\theta_{b} & \in\left[\theta_{b r}^{\prime}+\pi+\beta, \theta_{b r}^{\prime}+\pi+\beta+2 \alpha_{c}\right], \\
\phi_{b} & \in\left[\phi_{b s}^{\prime}+\pi+\beta-2 \alpha_{o}, \phi_{b s}^{\prime}+\pi+\beta\right] .
\end{aligned}
$$

These limits will be used to determine the number of rollers seated on the two sprockets. Expressing the angels to span $b$ in terms of the angles to span $a$ and integer increments of $2 \alpha$ gives

$$
\begin{aligned}
\theta_{b} & =\theta_{a}+2 \alpha_{c} \hat{n}_{c}, \\
\phi_{b} & =\phi_{a}+2 \alpha_{o} \check{n}_{o}-2 h \alpha_{o},
\end{aligned}
$$


where the function $h$ ensures that $\phi_{b}$ decrease continuously, as $\phi_{a}$ jumps discontinuously when a roller is released from the driven sprocket at $\phi_{a r}$. In (8)-(9) the total number of sprocket seating curves has been split into being either free or occupied by a chain roller, i.e.

$$
n=\check{n}+\hat{n}, \quad \check{n}, \hat{n} \in \mathbb{N},
$$

where the number of seating curves with a roller seated is $\check{n}$ and the number of seating curves with no roller seated is $\hat{n}$. Substituting (8) into (6) gives the number of free seating curves on the driver sprocket:

$$
\frac{\theta_{b r}^{\prime}-\theta_{a}+\pi+\beta}{2 \alpha_{c}} \leq \hat{n}_{c} \leq \frac{\theta_{b r}^{\prime}-\theta_{a}+\pi+\beta}{2 \alpha_{c}}+1 .
$$

Similarly, by substituting (9) into (7) the number of rollers seated on the driven sprocket can be determined from

$$
\frac{\phi_{b s}^{\prime}-\phi_{a}+\pi+\beta}{2 \alpha_{c}}-1+h \leq \check{n}_{o} \leq \frac{\phi_{b s}^{\prime}-\phi_{a}+\pi+\beta}{2 \alpha_{c}}+h .
$$

With $\hat{n}_{c}$ determined from (11), the contact angle to the lower span at the driver sprocket can be determined from (8). The contact angle to the lower span at the driven sprocket can be determined from (9) with $\check{n}_{O}$ determined from (12) and $h$ given in (2). The total wrapping length of the chain is then

$$
L=l_{a}+l_{b}+\left(\check{n}_{c}+\check{n}_{o}\right) p
$$

with $l_{a}$ given by (2), $\check{n}_{c}$ by (10) and the lower span length calculated from

$$
l_{b}=\sqrt{\left(x_{c}+R_{c} \cos \theta_{b}-R_{o} \cos \phi_{b}\right)^{2}+\left(y_{c}+R_{c} \sin \theta_{b}-R_{o} \sin \phi_{b}\right)^{2}} .
$$

In summary, the total length $L$ of the chain wrapping around both sprockets was determined by assuming that only span $b$ could deform. Because the lower span length varies as the sprockets rotate, it has been shown that a 
roller chain drive with tight spans must in general include compliant components to function. To improve the quantitative estimate of the wrapping length variation, the deformation of the other chain drive components should be included, i.e. the deformation of the both spans, and the deformation of chains wrapped on elastic sprockets $[33,34,35]$. The above analysis serves as an initial investigation of this effect of polygonal action, which has not been treated previously. Lastly, the kinematic configuration of all the chain drive components determined when the wrapping length equals an integer number of chain pitches is exact. This is useful for initializing e.g. multi-body simulations of roller chain drives.

\section{Example results of the kinematic analysis}

Twelve different chain drive configurations are used to present results for the calculation of the wrapping length, with ID and properties as listed in Table 1. For a given pitch length $p$, number of teeth $n_{c}, n_{o}$, span length given by $N$ and pitch fraction $f$, the sprocket center positions can be found from (1). The tight span length $l_{a}$ can be determined from (2), and the total number of links in the chain is given by $M$. Calculated values for the minimum, mean and maximum wrapping length obtained from (13) are presented, all nondimensionalized with the pitch $p$. Configuration $C_{1}$ has been subject to experimental measurements of "angular displacement error" [5] as well as kinematic studies of driven sprocket angular motion [7]. Note from Table 1, that if both chain spans are to be in tension for a given configuration, the pitch fraction $f$ cannot be chosen freely.

Figure 2 shows how the wrapping length varies during one tooth period for the configurations in Table 1. In general, the magnitude of the wrapping 
Table 1: Chain drive configurations

\begin{tabular}{ccccccccc}
\hline ID & $n_{c}$ & $n_{o}$ & $N$ & $M$ & $f$ & $L_{\min } / p$ & $L_{\text {mean }} / p$ & $L_{\text {max }} / p$ \\
\hline$A_{1}$ & 6 & 12 & 4 & 20 & 0.3325 & 20.0020 & 20.0200 & 20.0411 \\
$A_{2}$ & & & 8 & 28 & 0.4066 & 28.0027 & 28.0162 & 28.0329 \\
$A_{3}$ & & & 16 & 44 & 0.4516 & 44.0046 & 44.0130 & 44.0236 \\
\hline$B_{1}$ & 12 & 12 & 8 & 31 & 0.5015 & 31.0030 & 31.0032 & 31.0034 \\
$B_{2}$ & & & 16 & 46 & 0.0024 & 46.0048 & 46.0048 & 46.0048 \\
$B_{3}$ & & & 24 & 63 & 0.5032 & 63.0064 & 63.0065 & 63.0066 \\
\hline$C_{1}$ & 12 & 18 & 11 & 40 & 0.4302 & 40.0040 & 40.0091 & 40.0149 \\
$C_{2}$ & & & 22 & 62 & 0.4655 & 62.0066 & 62.0100 & 62.0138 \\
$C_{3}$ & & & 33 & 84 & 0.4786 & 84.0085 & 84.0110 & 84.0138 \\
\hline$D_{1}$ & 24 & 32 & 16 & 63 & 0.4106 & 63.0064 & 63.0079 & 63.0094 \\
$D_{2}$ & & & 24 & 79 & 0.4408 & 79.0080 & 79.0095 & 79.0112 \\
$D_{3}$ & & & 32 & 95 & 0.4569 & 95.0097 & 95.0111 & 95.0126 \\
\hline
\end{tabular}

length variation is seen to decrease as the number of sprocket teeth and span length is increased. For the special configuration $B$ where $n_{c} / n_{o}=1$, it is seen from Figure 2(b) that the length variation decreases significantly, and vanishes completely for configuration $B_{2}$, where the number of links $M$ equals an even number and $f \simeq 0$. Except for configurations $B$, the wrapping length variation resembles a harmonic function, albeit the variations are not completely symmetric. A time-varying wrapping length demonstrates that a chain drive with tight spans must generally include compliant components to function. 

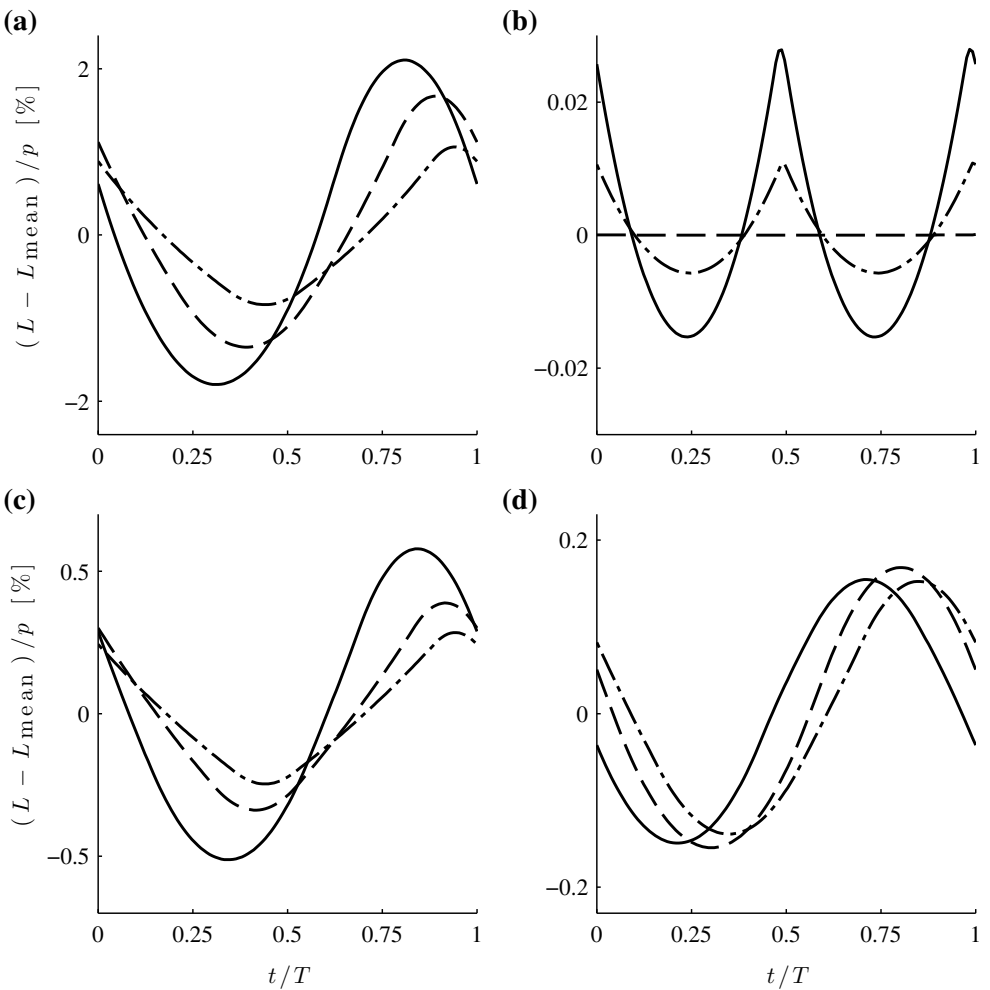

d)

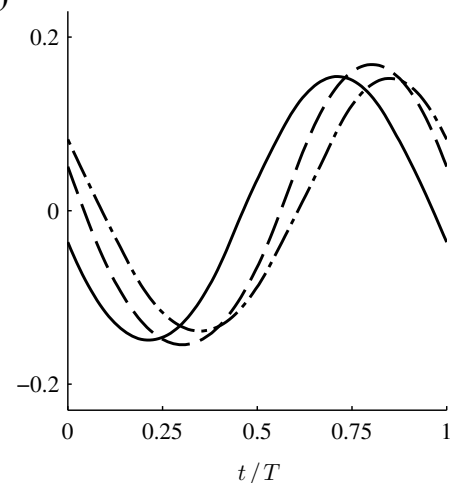

Figure 2: Chain wrapping length variation $L-L_{\text {mean }}$ normalized by pitch $p$ for one tooth period. Configurations $A-D$ are shown in figures (a)-(d), respectively. The line types (-), $(--)$ and $(-\cdot-)$ identify the, short, medium and long span lengths, respectively.

\section{Wrap length comparison with multi body simulation}

The kinematic analysis is based on a simplified model of a chain drive and the results describe the motion when deformations, clearances, dissipation and dynamics are neglected. In order to access the potential similarity with chain drives in operation, the kinematic results are compared with results from multibody simulations, which includes both greater detail of the chain drive geometry and dynamic effects. The multibody simulations are carried out using a simulation program developed by Pedersen et al. [8], which 
includes a detailed modeling of the tooth geometry and the flexible contact between teeth and chain rollers [9].

In the multibody model, the mass of the chain is assumed to be lumped at the roller locations, and the rotational inertia of the rollers about their centre of gravity is ignored. Springs and dampers with constant stiffness and damping coefficients model the links. Clearances between pin and bushing are neglected. Tooth geometry in the simulation program used to produce the results presented here is Type II per ASA B29.1-1950. For this comparison the chain drive configuration $C_{1}$ is used, which has also been analyzed experimentally [5]. Full details of the simulation parameters are comprehensive to present and only the main dynamic parameters are given: Chain pitch $0.0254 \mathrm{~m}$, link stiffness $0.221 \mathrm{GN} / \mathrm{m}$, link mass $0.066 \mathrm{~kg}$, link damping $0.01 \mathrm{Ns} / \mathrm{m}$, driven sprocket rotational inertia $0.046 \mathrm{kgm}^{2}$ and rotational damping $0.1 \mathrm{Nms} / \mathrm{rad}$. The driver angular velocity is constant at 10,100 and $300 \mathrm{rpm}$, and the simulations show no significant vibratory response of the chain spans or driven sprocket at these driver velocities.

Analytical predictions of the wrapping length given by (13) are compared to the results of multibody simulation and presented in Figure 3. It is seen that the minimum wrapping length predicted by the kinematic analysis is in excellent agreement with the simulation results. The wrapping length variation shows resemblance to harmonic functions, and the simulation and analytical results are in very good qualitative agreement. Quantitatively, the kinematic analysis predicts an amplitude of the wrapping length which is about a two times larger than what is observed in the simulation results. One reason for this could be that the multibody model allows for indentation of the rollers into the sprockets. Finally, it is seen that the phenomena is not limited to quasi static (low speed) operation of the chain drive, as 
the simulation results for 10, 100 and $300 \mathrm{rpm}$ all shows a tooth periodic variation of the wrapping length. As the driver velocity increases there is seen to be a slight increase in the minimum wrapping length, and one explanation for this could be an increased axial tension of the chain due to centrifugal effects.

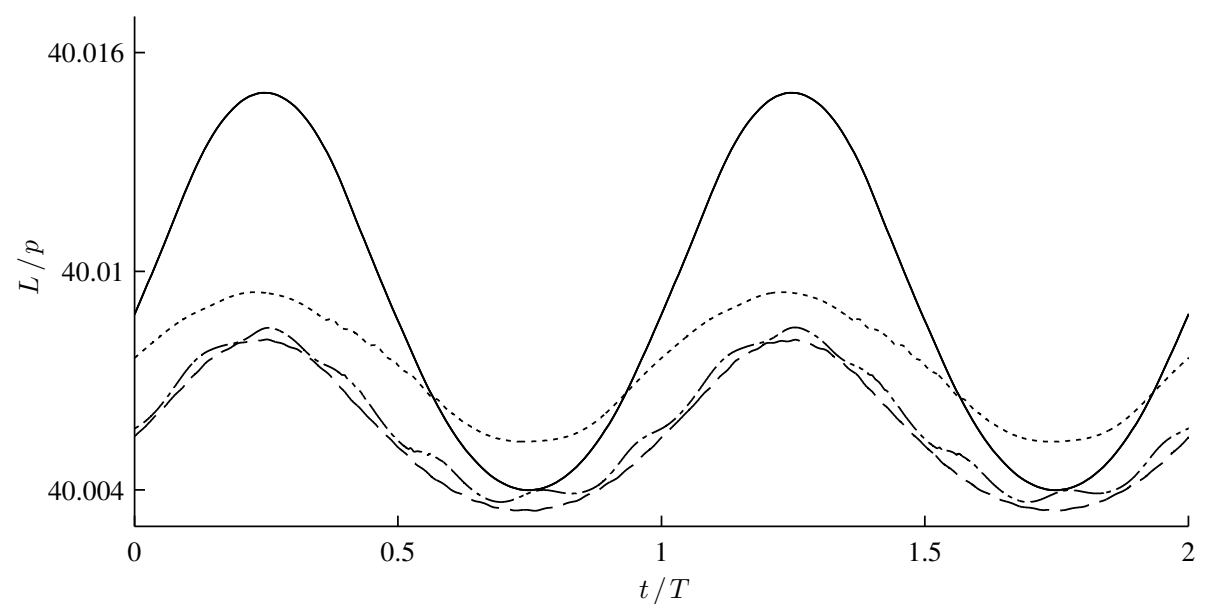

Figure 3: Normalized chain wrapping length $L / p$ for configuration $C_{1}$ for two tooth periods. Results in broken lines $(--),(-\cdot-)$ and $(\cdots)$ identify results from multibody simulation at a driver speed of 10, 100 and $300 \mathrm{rpm}$, respectively. The analytical prediction of the wrapping length is independent of the driver speed and is shown with a solid line $(-)$.

During installation of real chain drives, i.e. when the drive is stationary and unloaded, the span tension will depend on the angular position of the driver sprocket. During operation the axial force is then expected to vary periodically with the tooth frequency. The effect appears here for chain drives with only two sprockets, but is also expected for chain drive configurations with more sprockets and spans, and should also not be limited to low-speed chain drives. We hypothesize that multiple sprockets can be positioned to either attenuate or amplify the effect of a variable wrapping length. If this 
is the case, careful positioning of e.g. a tensioner sprocket could possibly neutralize the effect of a variable wrapping length, and allow for a constant span tension even for chain drive configurations where the sprockets have few teeth.

\section{Dynamic modeling and analysis}

First a model is derived which is capable of representing the coupling between the chain span transverse vibrations with the driven sprocket angular vibration. Then we present approximate solutions for the longitudinal displacements, and perform a mode shape expansion leading to coupled equations of motion of the tight span, the slack span and the driven sprocket. This resulting 3-DOF model is analyzed using the method of multiple scales, and three example results are presented.

The tight- and slack chain spans are assumed to have axial tension $P, \tilde{P}>$ 0 , respectively, thereby providing elastic coupling across the driven sprocket. In real applications of roller chain drives such as low speed marine engines, the roller chain is pretensioned in this way in order to minimize chain span transverse vibrations. Pretension is achieved by the positioning of a fixed tightener sprocket, ensuring that all chain spans in the drive are in positive tension. However, the choice of pretension giving the optimal vibration suppression at various operating conditions is not well understood, and in some designs guide-bars must be applied to limit transverse vibrations.

In roller chain drives both ends of the chain spans are subjected to transverse excitation from chain and sprocket meshing. Axial excitation of the chain span coming from polygonal action, non-uniform motion of the driver sprocket, and external loading of the driven sprocket will be included. 
Throughout the modeling and analysis we identify prescribed external forcing to be included in the multiple scales analysis by a star ( )*. Whenever possible, we do not present equations for the slack span, as for $\tilde{P}>0$ they are equivalent to equations for the tight span. Parameters and variables for the slack span are identified by a tilde $\left(^{\sim}\right)$, and their definitions are equivalent to the similar variables for the tight span without a tilde.

\subsection{Dynamic model}

A simple model consisting of two sprockets is considered, with gravity ignored. The local coordinate systems and definitions of displacements are

shown in Figure 4. The dependent variables $U, \tilde{U}, \hat{W}, \hat{\tilde{W}}$ and $\theta_{1}$ are the displacements measured at a steady state of operation, i.e. the particular solution of the forced response, calculated when the mean of the input torque $M_{2}$ equals the mean of the output torque $M_{1}$.

The motion of the driving sprocket is assumed to be given, i.e. $\theta_{2}^{*}$ is a known function of time. This represents the case where angular motion of e.g. a crankshaft is given, and the crankshaft kinematically forces the chain drive, as is relevant with combustion engines, where the chain drive powers auxiliary equipment requiring only a small fraction of the power required at the main output. For example with ship propulsion engines, the chain drive powering the hydraulic pumps is driven directly by the crankshaft, and consumes only about $1 \%$ of the power required for driving the propulsion propeller.

At steady state the input torque balances the output torque, such that there is no net transient acceleration and change of velocity of the drive. This is equivalent to the chain drive operating at nominal angular velocity [24]. Assuming the initial total span (pre)tension $P_{\text {tot }}=P_{\text {pre }}+\tilde{P}_{\text {pre }}$ at zero 


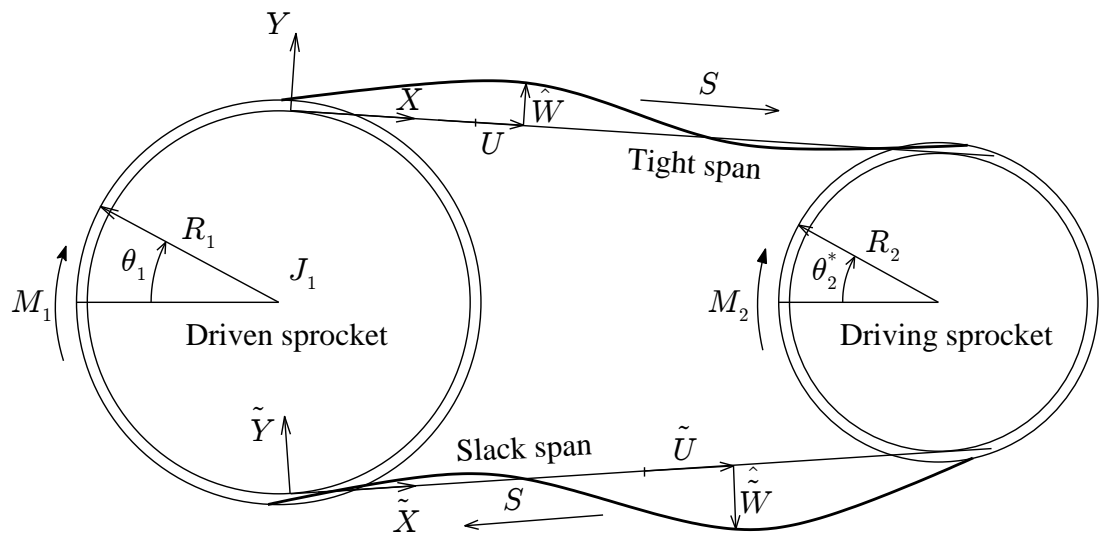

Figure 4: Mechanical model of a chain drive system with two sprockets

external load and zero angular velocity to be known, as well as the nominal span velocity $S$ and input torque $\hat{M}_{2}$, the longitudinal forces at steady state operation are, for the upper and lower spans

$$
\begin{aligned}
& P_{0}=\frac{1}{2}\left(P_{\text {tot }}+\hat{M}_{1} / R_{1}\right), \\
& \tilde{P}_{0}=\frac{1}{2}\left(P_{\text {tot }}-\hat{M}_{1} / R_{1}\right) .
\end{aligned}
$$

The output torque $\hat{M}_{1}$ can me modeled as comprised of a constant (brake) load $\hat{f}_{1}$, rotational viscous damping $\hat{d}_{1}$, and a time dependent torque $\hat{M}_{1}^{*}(t)$, where $t$ is time

$$
\hat{M}_{1}=\hat{d}_{1} S / R_{1}+R_{1} \hat{f}_{1}+\hat{M}_{1}^{*}
$$

For $\hat{M}_{1}^{*}=0$ (15)-(16) with (17) demonstrate how the nominal tension of the chain spans varies with initial pretension, operating speed, and constant external load. Centrifugal forces are not included in this model, which could be relevant for high-speed roller chain drives, and we note that chain wear corresponds to a reduction of $P_{\text {tot }}$. 
In the following section, the equations of motion for the chain spans are derived using Hamiltons principle. For the driven sprocket, the equation of motion will be derived using Newtons 2nd law. The governing equations are formulated such that the driven sprocket motion couples the tight and slack span motion through their boundary conditions.

\subsection{Equation of motion for the chain spans}

The roller chain is modeled as an axially moving string supported by moving boundaries, as illustrated in Figure 5. The coordinate system $X, Y$ is inertial and the moving string endpoint displacements are prescribed from the axial positions $X_{1}(T)$ and $X_{2}(T)$. In a chain drive, the chain span travels in the axial direction with the span endpoint constituted by the rollers seated on the rotating sprocket which has a neighboring roller in the free span. As the sprockets rotate, chain links recurrently enter and leave the span. Therefore the positions of the span endpoints from which the displacements are prescribed moves instantly at these events cf. Figure 1. Consequently, the amount of string material within the boundaries varies, as we see from the periodically varying span length given by (2) for the tight span. To model this, it is necessary to allow the position from which the span endpoint displacements are prescribed from to be functions of time; $X_{1}(T)$ and $X_{2}(T)$ models this. Between the seating and release events, the distance $X_{2}(T)-X_{1}(T)$ equals the span length, and the boundaries move in the positive x-direction at nominal speed $S \geq 0$.

From the moving span endpoint axial positions $X_{1}, X_{2}$, we denote the span endpoint longitudinal displacements by $U_{1}(T), U_{2}(T)$, respectively. In the transverse direction, the string endpoint displacements are given by $Y_{1}(T), Y_{2}(T)$. 


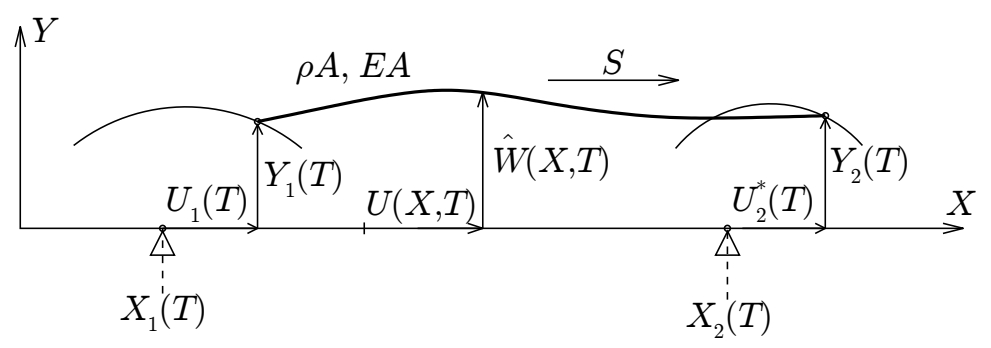

Figure 5: Axially moving chain span supported by moving boundaries

The string is assumed to have constant axial stiffness $E A$ and mass per unit length $\rho A$, and is subjected to axial tension $P>0$. Longitudinal and transverse displacements of the string are denoted by $U(X, T)$ and $\hat{W}(X, T)$, respectively, while out of plane motion is not considered. Both longitudinal and transverse displacements is possible at the moving span endpoints, i.e. the boundary conditions are inhomogeneous:

$$
\begin{aligned}
& U\left(X_{1}(T), T\right)=U_{1}(T), \quad U\left(X_{2}(T), T\right)=U_{2}^{*}(T) \\
& \hat{W}\left(X_{1}(T), T\right)=Y_{1}(T), \quad \hat{W}\left(X_{2}(T), T\right)=Y_{2}(T) \text {. }
\end{aligned}
$$

Kinematic forcing from the angular motion of the driver sprocket is assumed prescribed and identified by a star, $U_{2}^{*}$.

The axial and transverse velocity components are $U_{,_{T}}+S\left(1+U,_{X}\right)$ and $\hat{W}_{{ }_{T}}-S \hat{W},_{X}$, respectively. With this, the kinetic energy of the string is

$$
K(T)=\frac{\rho A}{2} \int_{X_{1}(T)}^{X_{2}(T)}\left(\left(U,_{T}+S(1+U, X)\right)^{2}+\left(\hat{W},_{T}-S \hat{W},{ }_{X}\right)^{2}\right) d X
$$

Since $X_{2}(T)-X_{1}(T)$ is not constant for all $T$, the amount of string material within the boundaries varies, and we can investigate the effect of a chain span having a time-varying length. When $P_{0}$ is the mean over time of the axial force $P$, the mean axial strain is $e_{0}=P_{0} / E A$, and the total strain is 
$e_{0}+e(X, T)$. The approximate nonlinear measure for the dynamical strain is $[15]$ :

$$
e(X, T)=U,{ }_{X}+\frac{1}{2} \hat{W}, X
$$

Using this, the potential energy associated with elastic string deformation is

$$
V(T)=\frac{E A}{2} \int_{X_{1}(T)}^{X_{2}(T)}\left(\frac{P_{0}}{E A}+U, X+\frac{1}{2} \hat{W}, \underset{X}{2}\right) d X,
$$

where $K(T)$ and $V(T)$ are most accurate when $\left|\hat{W},{ }_{X}\right|=|U, X|=O(\varepsilon)$, $\varepsilon \ll 1$ and $U=O\left(\hat{W}^{2}\right)$, such that nonlinear strain deriving from $U$ is negligible. The equations of motion and the associated boundary conditions can be derived using Hamilton's principle,

$$
\delta \int_{T_{1}}^{T_{2}}(K-V) d T=0 .
$$

By specifying the motion at the boundaries, and requiring the virtual displacements to be kinematically admissible, the displacement variations at the boundaries where motion is prescribed is zero; this ensures that the virtual work done at either boundary is zero [36]. The actual work done by the boundaries is not zero, and not required to be [37]. The total mechanical energy is not constant, even for fixed boundary conditions [38]. But the application of Hamiltons principle presupposes the applied forces to be derivable from a potential; it does not require constant energy. Non-constant energy is usually associated with damping (or external non-potential forces), but there are no dissipative forces in the system so far, and the equations of motion can be derived using (23).

Since the limits of integration in (20) and (22) are time dependent, the usual process of deriving the governing equations [10] using integrations by parts is not possible, and the use of Leibniz rule for differentiation of 
integrals is required, as in [39]. This gives:

$$
\begin{gathered}
\rho A\left(U,_{T T}+2 S U,,_{X T}+S^{2} U,_{X X}\right)-E A\left(U,_{X}+\frac{1}{2} \hat{W},_{X}^{2}\right),{ }_{X}=0, \\
\rho A\left(\hat{W},_{T T}+2 S \hat{W},_{X T}+S^{2} \hat{W},_{X X}\right)-E A\left(\hat{W},_{X}\left(U,_{X}+\frac{1}{2} \hat{W},_{X}^{2}+\frac{P_{0}}{E A}\right)\right),,_{X}=0,
\end{gathered}
$$

with boundary conditions given by (18)-(19). The functions $U_{1}(T)$ and $U_{2}^{*}(T)$ relates to the non-constant angular motion of the driven and driver sprockets, respectively; $U_{2}^{*}(T)$ is assumed to be given, and $U_{1}(T)$ will be formulated in terms of the angular displacements of the driven sprocket in section 5.5. Equations (24)-(25) are non-dimensionalized with all lengths measured relative to the length $l$,

$$
\left(x, u, \hat{w}, x_{1}, x_{2}, y_{1}, y_{2}\right)=\frac{\left(X, U, \hat{W}, X_{1}, X_{2}, Y_{1}, Y_{2}\right)}{l},
$$

where $l=|O C|$, and time is non-dimensionalized by the fundamental frequency of a fixed-fixed string of length $l$ under constant axial tension $P_{t}$,

$$
t=\sqrt{\frac{P_{t}}{\rho A l^{2}}} T .
$$

Here we do not specify $P_{t}$, as the convenient choice for $P_{t}$ depends on the object of the analysis; e.g. engine builders usually normalize to the driver frequency, in which case $P_{t}$ can be chosen accordingly. The corresponding non-dimensional equation of motion for the transverse motions $\hat{w}$ of the tight span becomes

$$
\hat{w}_{t t}+2 s \hat{w},{ }_{x t}+s^{2} \hat{w},_{x x}-\alpha\left(\hat{w},_{x}\left(u,_{x}+\frac{1}{2} \hat{w}_{, x}^{2}+\gamma\right)\right),_{x}=0,
$$

while longitudinal motions $u$ of the tight span are governed by

$$
u, t t+2 s u,_{x t}+s^{2} u,_{x x}-\alpha\left(u,_{x}+\frac{1}{2} \hat{w},_{x}^{2}\right),_{x}=0,
$$


where dimensionless parameters have been introduced as

$$
s=\frac{S}{\sqrt{\frac{P_{t}}{\rho A}}}, \alpha=\frac{E A}{P_{t}}, \gamma=\frac{P_{0}}{E A}, \tilde{\gamma}=\frac{\tilde{P}_{0}}{E A} .
$$

Note that, if $P_{t}=P$, then $\alpha \gamma=1$ and $\alpha \tilde{\gamma}=\tilde{P} / P$. The non-dimensional boundary conditions are

$$
\begin{array}{ll}
\hat{w}\left(x_{1}(t), t\right)=y_{1}(t), & \hat{w}\left(x_{2}(t), t\right)=y_{2}(t), \\
u\left(x_{1}(t), t\right)=u_{1}(t), & u\left(x_{2}(t), t\right)=u_{2}^{*}(t),
\end{array}
$$

where $u_{1}$ depends on the driven sprocket angular displacements and $u_{2}^{*}$ is given by the driving sprocket angular displacements.

\subsection{Solution for the longitudinal displacement and axial tension}

The transverse wave speed for a roller chain under axial tension $P_{0}$ and mass per unit length $\rho A$ is $c_{w}=\sqrt{P_{0} / \rho A}$. The natural frequencies of a stationary $(s=0)$ fixed-fixed string with length $l$ are $n c_{w} / 2 l, n=1,2, \ldots$. Assuming $E A$ and $\rho A$ to be constant, the longitudinal wave speed is $c_{u}=$ $E A / \rho A$. The analysis is restricted to roller chain spans operating under low or moderate loads, and with a maximum allowable tension $P_{\max }$ being small compared to the axial stiffness $E A$. The longitudinal waves propagate much faster than the lower transverse waves, and the influence of longitudinal inertia is small. Under this assumption the axial force is only time dependent, i.e. $N,_{x}=0$.

The instantaneous axial tension $N$ can be identified from (28) as the coefficient to $\hat{w}{ }_{x x}$ :

$$
N(x, t)=\alpha\left(u,_{x}+\frac{1}{2} \hat{w},_{x}^{2}+\gamma\right)-s^{2} .
$$


Using $N,_{x}=0$ gives

$$
\left(u,_{x}+\frac{1}{2} \hat{w},_{x}^{2}\right)_{, x}=0,
$$

which shows that the strain is also constant along the axial direction, $e,_{x}=$ 0. Integrating (21) with the boundary conditions (32), the axial dynamic dynamic strain becomes:

$$
e(t)=\frac{u_{2}^{*}(t)-u_{1}(t)+\frac{1}{2} \int_{x_{1}(t)}^{x_{2}(t)} \hat{w}_{x}^{2} d x}{x_{2}(t)-x_{1}(t)} .
$$

Integrating (21) from $x_{1}(t)$ to $x$ gives the solution for the axial displacement

$$
u(x, t)=u_{1}(t)+e(t)\left(x-x_{1}(t)\right)-\frac{1}{2} \int_{x_{1}(t)}^{x} \hat{w},{ }_{x}^{2} d x .
$$

Which shows how $u$ depends nonlinearly on the transverse motion $\hat{w},{ }_{x}$. The axial force is then given by

$$
N(t)=\alpha(e(t)+\gamma)-s^{2},
$$

which becomes useful when deriving the equation of motion for the driven sprocket.

\subsection{Obtaining homogeneous boundary conditions for the transverse motion}

To obtain homogeneous boundary conditions we employ a linear transformation

$$
\hat{w}(x, t)=w(x, t)+y_{1}(t)(1-x)+y_{2}(t) x .
$$

Substituting into (28) and (35) for the dynamic strain, the equation of transverse motion becomes

$w, t t+2 s w, x t-\left(\alpha \gamma-s^{2}\right) w,_{x x}-\alpha e(t) w,_{x x}=-y, t t(1-x)-y_{2, t t}-2 s\left(y_{2, t}-y_{1, t}\right)$, 
with boundary conditions

$$
w\left(x_{1}(t), t\right)=0, w\left(x_{2}(t), t\right)=0 .
$$

Although homogeneous, the boundary conditions are specified from a moving position. For roller chain drives the change in span endpoint position is small compared to the span length; it is therefore reasonable to limit the analysis to small axial variations of the support positions. They are assumed to be of the form

$$
x_{1}(t)=\varepsilon \Delta x_{1}(t), x_{2}(t)=1+\varepsilon \Delta x_{2}(t),
$$

where $\varepsilon \ll 1$ denotes smallness of terms. Taylor expanding the boundary conditions (40) around $x=0$ and $x=1$ gives

$$
\begin{aligned}
& w\left(x_{1}(t), t\right)=w(0, t)+\varepsilon \Delta x_{1}(t) w(0, t),{ }_{x}+O\left(\varepsilon^{2}\right) \\
& w\left(x_{2}(t), t\right)=w(1, t)+\varepsilon \Delta x_{2}(t) w(1, t),{ }_{x}+O\left(\varepsilon^{2}\right) .
\end{aligned}
$$

When deriving the kinetic and potential energies it was used that $\left|U_{,_{x}}\right|=$ $\left|\hat{W},_{x}\right|=O(\varepsilon)$, which is fulfilled when $\left|w,_{x}\right|=O(\varepsilon)$. Then (42)-(43) reduce to

$$
\begin{aligned}
& w\left(x_{1}(t), t\right)=w(0, t)+O\left(\varepsilon^{2}\right), \\
& w\left(x_{2}(t), t\right)=w(1, t)+O\left(\varepsilon^{2}\right) .
\end{aligned}
$$

For roller chain drives the transverse displacements of the chain span endpoints is also small compared to the span length, and it is reasonable to specify the displacements of the boundaries to satisfy $\left|y_{2}-y_{1}\right|=O(\varepsilon)$ and $\left|x_{1}-x_{2}\right|=O(1)$. Then the dynamic strain (35) reduces to

$$
e(t)=u_{2}^{*}-u_{1}+\frac{1}{2} \int_{0}^{1} w_{x}^{2} d x+O\left(\varepsilon^{2}\right),
$$


and the equation of motion for the transverse motion of the tight span becomes

$$
\begin{aligned}
w, t t+2 s w,_{x t}-\left(\alpha \gamma-s^{2}\right) w,_{x x}-\varepsilon \alpha\left(u_{2}^{*}-u_{1}+\frac{1}{2} \int_{0}^{1} w_{,_{x}}^{2} d x\right) w,_{x x} \\
=-y_{1, t t}(1-x)-y_{2, t t} x-2 s\left(y_{2, t}-y_{1, t}\right)+O\left(\varepsilon^{2}\right) .
\end{aligned}
$$

with boundary conditions

$$
\begin{aligned}
& w(0, t)=0+O\left(\varepsilon^{2}\right), \\
& w(1, t)=0+O\left(\varepsilon^{2}\right) .
\end{aligned}
$$

Thus, the effect of boundary positions moving in the axial and transverse direction by a small amount $O(\varepsilon)$ leads to a second order effect in the equation of motion and boundary conditions for transverse vibrations of the chain span.

\subsection{Driven sprocket equation of motion}

For the driven sprocket it is assumed that the sprocket center is fixed such that only rotation around the sprocket center is possible. The equation of motion can be obtained using Newton's second law, which gives

$$
\hat{J}_{1} \theta_{1}, T T+\hat{d}_{1} \theta_{1}, T=R_{1}(N-\tilde{N})+\hat{M}_{1},
$$

where $\hat{J}_{1}$ is the mass moment of inertia for the driven sprocket and attached machinery, $\hat{d}_{1}$ is the coefficient of viscous rotational damping of sprocket motion, $\hat{M}_{1}$, is the externally applied torque, and $N, \tilde{N}$ are the axial reaction forces from the upper and lower chain span, respectively. Assuming a purely kinematic relationship between the sprocket angular displacements $\theta_{1,2^{*}}$ and the span endpoint longitudinal displacements $u_{1,2^{*}}$ allows us to write

$$
\theta_{1}=\frac{l}{R_{1}} u_{1}, \quad \theta_{2}^{*}=\frac{l}{R_{2}} u_{2}^{*}
$$


Inserting this, and using (27) and (33) gives

$$
J u_{1, t t}+d u_{1, t}=\alpha\left(u,_{x}+\frac{1}{2} \hat{w}_{x^{2}}^{2}+\gamma-\frac{s^{2}}{\alpha}\right)-\alpha\left(\tilde{u},_{x}+\frac{1}{2} \hat{\tilde{w}}_{x}^{2}+\tilde{\gamma}-\frac{s^{2}}{\alpha}\right)+M_{1},
$$

where

$$
J=\frac{\hat{J}_{1}}{\rho A l R_{1}^{2}}, d=\frac{\hat{d}_{1}}{R_{1}^{2} \sqrt{\rho A P_{t}}}, M_{1}=\frac{\hat{M}_{1}}{R_{1} P_{t}} .
$$

When the mean axial forces $P$ and $\tilde{P}$ in (30) are chosen such that they balance the nominal output torque (17), (52) reduces to

$$
J u_{1, t t}+d u_{1, t}=\alpha\left(u,_{x}+\frac{1}{2} \hat{w}, x^{2}\right)-\alpha\left(\tilde{u},_{x}+\frac{1}{2} \hat{\tilde{w}}_{x}^{2}\right)+M_{1}^{*}(t),
$$

where $M_{1}^{*}(t)$ is the time dependent driven sprocket torque with zero mean. The two terms multiplied by $\alpha$ can be recognized to be the dynamic axial strains of the upper and lower spans. Under the already made assumptions of $e,{ }_{x}=0$ and small endpoint displacements these are given by (46). Assuming rigid connection of the upper and lower span across the sprocket, i.e.

$$
u_{1}=-\tilde{u}_{1}, u_{2}^{*}=-\tilde{u}_{2}^{*}
$$

and inserting the dynamic strains given by (46), using (55) to eliminate $\tilde{u}_{1}, \tilde{u}_{2},(54)$ becomes

$$
J u_{1, t t}+d u_{1, t}+2 \alpha u_{1}=2 \alpha u_{2}^{*}+\frac{\alpha}{2} \int_{0}^{1}\left(w,_{x}^{2}-\tilde{w}_{x}^{2}\right) d x+M_{1}^{*}(t) .
$$

This is an inhomogeneous ordinary second order differential equation for the driven sprocket angular displacements $u_{1}$ measured from steady state operating conditions. The equation is nonlinearly coupled to the upper and lower span transverse displacements $w$ and $\tilde{w}$. 


\subsection{Mode shape expansion}

Because the main focus is to study the coupling between the two spans and the driven sprocket it is chosen to use stationary mode shapes for the discretization of the equations governing the transverse vibrations of the string. As noted in the introduction, using complex mode shapes provides a better basis for estimating parametric instability. However, to simplify the algebra and produce results which are more easily interpreted, and still valid at lower speeds $s,(47)$ is discretized using the standard Galerkin method with test functions chosen as mode shapes for transverse vibrations of an axially non-moving uniform string:

$$
w(x, t)=\sum_{n=1}^{N} \xi_{n}(t) \phi_{n}(x), \quad \phi_{n}(x)=\sin n \pi x .
$$

Following the standard Galerkin procedure, the equation governing the modal amplitudes $\xi_{m}, m=1,2, \ldots, N$ becomes

$$
\begin{aligned}
\xi_{m, t t}+2 s C_{m n} \zeta_{m, t} & +\left(\alpha \gamma-s^{2}\right)(m \pi)^{2} \xi_{m}+\alpha(m \pi)^{2}\left(u_{2}^{*}-u_{1}+\frac{1}{4} \sum_{n=1}^{N} \xi_{n}^{2}(n \pi)^{2}\right) \xi_{m} \\
& =-\left(y_{1, t t}-2 s\left(y_{2}, t-y_{1}, t\right)\right) G_{m}+\left(y_{1, t t}-y_{2}, t t\right) E_{m},
\end{aligned}
$$

where

$$
C_{m n}=\frac{2 m n\left(1-(-1)^{m+n}\right)}{m^{2}+n^{2}}, G_{m}=\frac{2\left(1-(-1)^{m}\right)}{m \pi}, E_{m}=\frac{-2(-1)^{m}}{m \pi} .
$$

It is seen here that the non-linear term introduces modal coupling. The Coriolis term (with $C_{m n}$ ) will only be included with an expansion including an even and an odd mode.

\subsection{Single mode approximation}

For the purpose of this analysis, where there is modal coupling between the tight and slack span, only a single mode expansion will be used for 
each span. For a single $m$ mode, the equation for the corresponding modal coordinate becomes:

$$
\begin{aligned}
\xi_{m, t t}+2 \mu_{m} \xi_{m, t}+ & \omega_{m}^{2} \xi_{m}+\varepsilon \alpha_{m}\left(u_{2}^{*}-u_{1}+p_{o}^{*}(t)\right) \xi_{m}+\varepsilon \kappa_{m} \xi_{m}^{3} \\
& =-\left(y_{1, t t}-2 s\left(y_{2, t}-y_{1, t}\right)\right) G_{m}+\left(y_{1, t t}-y_{2}, t t\right) E_{m}
\end{aligned}
$$

where

$$
\omega_{m}^{2}=\left(\alpha \gamma-s^{2}\right)(m \pi)^{2}, \alpha_{m}=(m \pi)^{2} \alpha, \kappa_{m}=\frac{1}{4}(m \pi)^{4} \alpha,
$$

linear modal damping with coefficient $2 \mu_{m}$ has been added, and axially varying tension coming from the varying wrapping length as specified by the function $p_{o}^{*}(t)$ has been included.

\subsection{Modeling transverse excitation}

The span endpoint positions $y_{1}, y_{2}$ follows curves which resemble a cycloid. At the instant where a roller enters (or leaves) the chain span, the projections of the nominal tangential velocity $s$ in the $y$-direction changes discontinuously, cf. Figure 1. Therefore, the endpoint transverse velocities are non-smooth in time, and the span endpoint accelerations also jumps discontinuously in time, which leads to impulsive loading of the chain spans from meshing. The external non-dimensional modal forcing $f_{m}$ depends on the transverse velocity and acceleration of the span endpoints, and is given by the right hand side of $(60)$ :

$$
f_{m}(t)=-\left(y_{1, t t}-2 s\left(y_{2}, t-y_{1, t}\right)\right) G_{m}+\left(y_{1, t t}-y_{2}, t t\right) E_{m},
$$

where $G_{m}=0$ for $m$ even, while $E_{m} \neq 0$ for all $m$. To represent the impulsive loading at the span endpoints, the acceleration jumps are modeled 
using Dirac pulse trains,

$$
y_{1, t t}=\sum_{j=-\infty}^{\infty} Q_{1} \delta\left(t-j \tau_{o}\right), \quad y_{2}, t t=\sum_{j=-\infty}^{\infty} Q_{2} \delta\left(t-(\psi+j) \tau_{o}\right),
$$

where $\tau_{o}=\sqrt{P_{t} / \rho A l^{2}}\left(n_{2} f_{2}\right)^{-1}$ is the non-dimensional tooth period, $f_{2}$ is the driver frequency in $\mathrm{Hz}$, and $n_{2}$ the number of teeth on the driver sprocket. The acceleration jump at the driven sprocket $Q_{1}$ occurs at the instant where a roller is released from the sprocket. Similarly, the acceleration jump $Q_{2}$ occurs at the instant where a roller seats on the driver sprocket. The Dirac pulses of the two endpoint accelerations are separated in time by a phase $\psi$, which relates to the shaft center distance, specifically the pitch fraction $f[7]$. Assuming the span to remain straight, the relative velocities between two neighbouring rollers at the instant of seating and release, respectively, are given by

$$
Q_{1}=2 s \sin \alpha_{1}=\frac{s p}{R_{1}}, \quad Q_{2}=2 s \sin \alpha_{2}=\frac{s p}{R_{2}},
$$

where $\alpha_{1}, \alpha_{2}$ are the pitch angles, $R_{1}, R_{2}$ are the pitch circle radius, $p$ is the pitch length, and it has been used that $R_{1}=p /\left(2 \sin \alpha_{1}\right)$ and $R_{2}=$ $p /\left(2 \sin \alpha_{2}\right)$. The same relative velocities are used in [32] for the study of impact between a roller seating at the driver sprocket. It is seen from (64) that increased sprocket radius, shorter pitch length and lower velocities reduce the endpoint acceleration discontinuities. Assuming the acceleration terms to be dominating, the terms proportional to $s$ are neglected in (62), and the approximation of the modal forcing becomes

$$
f_{m}(t)=\left(E_{m}-G_{m}\right) \sum_{j=-\infty}^{\infty} Q_{1} \delta\left(t-j \tau_{o}\right)-E_{m} \sum_{j=-\infty}^{\infty} Q_{2} \delta\left(t-(\psi+j) \tau_{o}\right) .
$$


Since the forcing is time-periodic, it can be written as a (complex-valued) Fourier series,

$$
f_{m}(t)=\sum_{p=-\infty}^{\infty} k_{p} e^{i 2 \pi p t / \tau_{o}}
$$

where

$$
k_{p}=\frac{1}{\tau_{o}}\left(\left(E_{m}-G_{m}\right) Q_{1}-E_{m} Q_{2} e^{-i 2 \pi p \psi}\right) .
$$

The Fourier coefficients have the same value for all $p$, due to the properties of the Dirac pulse train. The coefficient $k_{p}$ is complex, due to the phase $\psi$ and forcing can be obtained as the real part of (66). For high-speed drives, the loading coming from $Q_{2}$ will be dominant, as intertial forces reduce the discontinuous velocity jump at the driven sprocket. The approximate forcing introduced here is suitable for the purpose of investigating the toothperiodic impulsive loading inherent to chain drives; shown here to lead to multi-frequency external transverse excitation.

\subsection{Multiple scales perturbation analysis}

The vibrations of the chain spans and the driven sprocket are governed by coupled non-linear equations. To analyze the dynamics of this system the Method of Multiple Scales is employed. This relies on the sorting of terms into orders of magnitude, and the results and solution procedure depends on this ordering. Here, aiming at identifying and analyzing possible resonance conditions, we assume damping, parametric coupling and excitation to be weak, as well as non-linearity, and the forcing on the chain spans from impact loading. Under these assumptions, with smallness of terms identified by a book keeping parameter $\varepsilon \ll 1$, the governing equations for transverse vibrations of the upper and lower span given by (60) with external forcing 
(66), and rotational vibrations of the driven sprocket (56) are:

$$
\begin{gathered}
\xi_{m, t t}+2 \varepsilon \mu_{m} \xi_{m, t}+\omega_{m}^{2} \xi_{m}+\varepsilon \alpha_{m}\left(u_{2}^{*}-u_{1}+p_{o}^{*}(t)\right) \xi_{m}+\varepsilon \kappa_{m} \xi_{m}^{3} \\
=\varepsilon \sum_{j=-\infty}^{\infty} k_{m} e^{i j \Omega_{o} t}, \\
\tilde{\xi}_{n, t t}+2 \varepsilon \tilde{\mu}_{n} \tilde{\xi}_{n, t}+\tilde{\omega}_{n}^{2} \tilde{\xi}_{m}+\varepsilon \tilde{\alpha}_{n}\left(u_{1}-u_{2}^{*}+p_{o}^{*}(t)\right) \tilde{\xi}_{n} \\
+\varepsilon \tilde{\kappa}_{n} \tilde{\xi}_{n}^{3}=\varepsilon \sum_{j=-\infty}^{\infty} \tilde{k}_{n} e^{i j \Omega_{o} t+\phi}, \\
u_{1, t t}+2 \varepsilon \mu_{\theta} u_{1, t}+\omega_{\theta}^{2} u_{1}+\varepsilon \tilde{\eta}_{n} \tilde{\xi}_{n}^{2}-\varepsilon \eta_{m} \xi_{m}^{2}=\varepsilon \omega_{\theta}^{2} u_{2}^{*}+\varepsilon M_{1}^{*} J^{-1},
\end{gathered}
$$

where (57) has been inserted in (56), $\Omega_{o}=2 \pi / \tau_{o}$, a phase $\phi \in\left[0, \tau_{o}\right]$ has been introduced between the upper and lower span external loading (which depends on the chain drive configuration), and

$$
2 \mu_{\theta}=\frac{d}{J}, \quad \omega_{\theta}=\sqrt{\frac{2 \alpha}{J}}, \quad \eta_{m}=\frac{\alpha(m \pi)^{2}}{4 J}, \quad \tilde{\eta}_{n}=\frac{\alpha(n \pi)^{2}}{4 J} .
$$

We seek solutions in the form of uniformly valid expansions:

$$
\begin{aligned}
& \xi_{m}(t)=q_{0}\left(T_{0}, T_{1}\right)+\varepsilon q_{1}\left(T_{0}, T_{1}\right)+O\left(\varepsilon^{2}\right), \\
& \tilde{\xi}_{n}(t)=\tilde{q}_{0}\left(T_{0}, T_{1}\right)+\varepsilon \tilde{q}_{1}\left(T_{0}, T_{1}\right)+O\left(\varepsilon^{2}\right), \\
& u_{1}(t)=\nu_{0}\left(T_{0}, T_{1}\right)+\varepsilon \nu_{1}\left(T_{0}, T_{1}\right)+O\left(\varepsilon^{2}\right),
\end{aligned}
$$

where $T_{0}=t$ and $T_{1}=\varepsilon t$ is the slow time. Substituting into (68)-(70) and equating to zero like powers of $\varepsilon$ gives, to order $\varepsilon^{0}$ :

$$
\begin{aligned}
& D_{0}^{2} q_{0}+\omega_{m}^{2} q_{0}=0, \\
& D_{0}^{2} \tilde{q}_{0}+\tilde{\omega}_{n}^{2} \tilde{q}_{0}=0, \\
& D_{0}^{2} \nu_{0}+\omega_{\theta}^{2} \nu_{0}=0,
\end{aligned}
$$


where $D_{i}^{i} \equiv \partial^{j} / \partial T_{i}^{j}$, and to order $\varepsilon^{1}$ :

$$
\begin{gathered}
D_{0}^{2} q_{1}+\omega_{m}^{2} q_{1}=-2 D_{0} D_{1} q_{0}-2 \mu_{m} D_{0} q_{0}+\alpha_{m}\left(\nu_{0}-u_{2}^{*}+p_{o}^{*}\left(T_{0}\right)\right) q_{0} \\
-\kappa_{m} q_{0}^{3}+\sum_{j=-\infty}^{\infty} k_{m} e^{i j \Omega_{o} t}, \quad(78) \\
D_{0}^{2} \tilde{q}_{1}+\tilde{\omega}_{n}^{2} \tilde{q}_{1}=-2 D_{0} D_{1} \tilde{q}_{0}-2 \tilde{\mu}_{n} D_{0} \tilde{q}_{0}+\tilde{\alpha}_{n}\left(u_{2}^{*}-\nu_{0}+p_{o}^{*}\left(T_{0}\right)\right) \tilde{q}_{0} \\
-\tilde{\kappa}_{n} \tilde{q}_{0}^{3}+\sum_{j=-\infty}^{\infty} \tilde{k}_{n} e^{i j \Omega_{o} t+\phi} \\
D_{0}^{2} \nu_{1}+\omega_{\theta}^{2} \nu_{1}=-2 D_{0} D_{1} \nu_{0}-2 \mu_{\theta} D_{0} \nu_{0}+\eta_{m} q_{0}^{2}-\tilde{\eta}_{n} \tilde{q}_{0}^{2}+\omega_{\theta}^{2} u_{2}^{*}+M_{1}^{*} / J
\end{gathered}
$$

The general solutions to (75)-(77) are

$$
\begin{aligned}
& q_{0}=A\left(T_{1}\right) e^{i \omega_{m} T_{+}}+c c \\
& \tilde{q}_{0}=\tilde{A}\left(T_{1}\right) e^{i \tilde{\omega}_{n} T_{+}}+c c \\
& \nu_{0}=V\left(T_{1}\right) e^{i \omega_{\theta} T_{0}}+c c
\end{aligned}
$$

where the unknown functions $A, \tilde{A}, V$ are complex functions of slow time to be determined, and $c c$ complex conjugates of preceeding terms.

So far, the parametric excitation $p_{o}^{*}(t)$, the kinematic forcing of the driver sprocket $u_{2}^{*}(t)$ and the external excitation of the driven sprocket $M_{1}^{*}(t)$ has been assumed to have a zero mean, but otherwise arbitrary. Assuming each of them to be mono-frequency harmonic,

$$
\begin{aligned}
p_{o}^{*} & =p_{0} \cos \left(\Omega_{0} T_{0}\right), \\
M_{1}^{*} / J & =p_{1} \cos \left(\Omega_{1} T_{0}\right), \\
u_{2}^{*} & =p_{2} \cos \left(\Omega_{2} T_{0}\right),
\end{aligned}
$$


and substituting (81)-(83) into (78)-(80), gives

$$
\begin{aligned}
& D_{0}^{2} q_{1}+\omega_{m}^{2} q_{1}= \frac{1}{2} k_{m}-\left(2 i \omega_{m} A^{\prime}+2 i \mu_{m} \omega_{m} A+3 \kappa_{m} A^{2} \bar{A}\right) e^{i \omega_{m} T_{0}} \\
&\left.+\alpha_{m}\left(A V e^{i\left(\omega_{m}+\omega_{\theta}\right) T_{0}}\right)+\bar{A} V e^{i\left(\omega_{\theta}-\omega_{m}\right) T_{0}}\right) \\
&-\frac{1}{2} \alpha_{m} p_{2}\left(A e^{i\left(\Omega_{2}+\omega_{m}\right) T_{0}}+\bar{A} e^{i\left(\Omega_{2}-\omega_{m}\right) T_{0}}\right) \\
&+\frac{1}{2} \alpha_{m} p_{0}\left(A e^{i\left(\Omega_{0}+\omega_{m}\right) T_{0}}+\bar{A} e^{i\left(\Omega_{0}-\omega_{m}\right) T_{0}}\right) \\
&-\kappa_{m} A^{3} e^{3 i \omega_{m} T_{0}}+\sum_{j=1}^{\infty} k_{m} e^{i j \Omega_{o} t}+c c
\end{aligned}
$$

$$
\begin{aligned}
D_{0}^{2} \tilde{q}_{1}+\tilde{\omega}_{n}^{2} \tilde{q}_{1}= & \frac{1}{2} \tilde{k}_{n}-\left(2 i \tilde{\omega}_{n} \tilde{A}^{\prime}+2 i \tilde{\mu}_{n} \tilde{\omega}_{n} \tilde{A}+3 \tilde{\kappa}_{n} \tilde{A}^{2} \tilde{\bar{A}}\right) e^{i \tilde{\omega}_{n} T_{0}} \\
& \left.-\tilde{\alpha}_{n}\left(\tilde{A} V e^{i\left(\tilde{\omega}_{n}+\omega_{\theta}\right) T_{0}}\right)+\tilde{\bar{A}} V e^{i\left(\omega_{\theta}-\tilde{\omega}_{n}\right) T_{0}}\right) \\
& +\frac{1}{2} \tilde{\alpha}_{n} p_{2}\left(\tilde{A} e^{i\left(\Omega_{2}+\tilde{\omega}_{n}\right) T_{0}}+\tilde{\bar{A}} e^{i\left(\Omega_{2}-\tilde{\omega}_{n}\right) T_{0}}\right) \\
& +\frac{1}{2} \tilde{\alpha}_{n} p_{0}\left(\tilde{A} e^{i\left(\Omega_{0}+\tilde{\omega}_{n}\right) T_{0}}+\tilde{\bar{A}} e^{i\left(\Omega_{0}-\tilde{\omega}_{n}\right) T_{0}}\right) \\
& -\tilde{\kappa}_{n} \tilde{A}^{3} e^{3 i \tilde{\omega}_{n} T_{0}}+\sum_{j=1}^{\infty} \tilde{k}_{n} e^{i\left(j \Omega_{o} t+\phi\right)}+c c
\end{aligned}
$$

$$
\begin{aligned}
D_{0}^{2} \nu_{1} & \left.+\omega_{\theta}^{2} \nu_{1}=\eta_{m} A \bar{A}-\eta_{n} \tilde{A} \tilde{\bar{A}}-\left(2 i \omega_{\theta}\right) V^{\prime}+2 i \mu_{\theta} \omega_{\theta} V\right) e^{i \omega_{\theta} T_{0}} \\
& +\eta_{m} A^{2} e^{i 2 \omega_{m} T_{0}}-\tilde{\eta}_{n} \tilde{A}^{2} e^{i 2 \tilde{\omega}_{n} T_{0}}+\frac{1}{2} \omega_{\theta}^{2} p_{2} e^{i \Omega_{2} T_{0}}+\frac{1}{2} p_{1} e^{i \Omega_{1} T_{0}}+c c
\end{aligned}
$$

The external sources of parametric excitation of the spans are from $p_{0}$ and $p_{2}$. When $p_{1}=0$ and $p_{0} \neq 0$ there will be parametric excitation of the spans, and the driven sprocket is only forced by span transverse vibration. When $p_{1} \neq 0$ or $p_{2} \neq 0$, there is parametric excitation of the spans and also direct excitation of the driven sprocket. To proceed with the solution of the coupled equations (87)-(89) it is necessary to identify resonant terms 
for each equation. For (87), certain right hand terms are resonant to the homogeneous part under conditions of:

External resonance: $j \Omega_{0} \approx \omega_{m}$,

Primary parametric resonance: $\Omega_{2} \approx 2 \omega_{m}$ and/or $\Omega_{0} \approx 2 \omega_{m}$,

Internal two-to-one resonance: $\omega_{\theta} \approx 2 \omega_{m}$.

Similarly for (88):

External resonance: $j \Omega_{0} \approx \tilde{\omega}_{n}$

Primary parametric resonance: $\Omega_{2} \approx 2 \tilde{\omega}_{n}$ and/or $\Omega_{0} \approx 2 \tilde{\omega}_{n}$,

Internal two-to-one resonance: $\omega_{\theta} \approx 2 \tilde{\omega}_{n}$,

and for (89):

External resonance: $\Omega_{2} \approx \omega_{\theta}$ and/or $\Omega_{1} \approx \omega_{\theta}$,

Primary parametric resonance: $\Omega_{2} \approx 2 \tilde{\omega}_{n}$ and/or $\Omega_{0} \approx 2 \tilde{\omega}_{n}$,

Internal two-to-one resonance: $2 \tilde{\omega}_{n} \approx \omega_{\theta}$ and/or $2 \omega_{m} \approx \omega_{\theta}$.

Thus, a multitude of resonance - and combined resonance cases exists, which involves one, two or all three degrees of freedom $q_{1}, \tilde{q}_{1}$, and $\nu_{1}$. Note that for e.g. the tight span motion $q_{1}$, primary parametric and external resonance coming from polygonal action ( $p_{0}$ and $k_{m}$ terms) cannot exist simultaneously, since primary parametric resonance requires $\Omega_{0} \approx \omega_{m}$, in which case the external forcing is has frequency $j \Omega_{0} \approx j 2 \omega_{m}$, i.e. non-resonant. Primary parametric resonance of both spans simultaneously is practically possible when there is no torque on the driven sprocket, as in the case of a guide or coupler sprocket; under these conditions the natural frequency of the two spans will be nearly identical, and coupling could occur across the 
driven sprocket if it is compliant. This case, as well as resonant transverse span excitation $\left(k_{m}\right.$ terms), possibly in combination with other resonant excitations, are left for future studies.

In the following we investigate three resonance cases which are of practical interest, but also demonstrate how the motions (de)couple in this model; primary parametric resonance of the tight span is treated first, then external resonance of the driven sprocket, and lastly, the two cases combined, i.e. primary parametric resonance of the tight span along with internal resonance of the driven sprocket.

\subsection{Primary parametric resonance of the tight span}

This example is relevant, since it demonstrates how decoupled transverse vibration of a single chain span, coming from a harmonic variation of axial tension leading to parametric resonance, could be realized in a real roller chain drive. We consider primary parametric resonance of the tight span $q_{1}$ coming from $p_{2}$. There are no other external or internal resonances, and the tight and slack span are detuned such that $\omega_{m}$ is away from $\tilde{\omega}_{n}$. For the external forcing coming from $p_{2}$, the nearness to primary parametric resonance of the tight span is quantified by a detuning parameter $\sigma_{2}$, such that

$$
\Omega_{2}=2 \omega_{m}+\varepsilon \sigma_{2},
$$

Inserting into (87)-(89) and requiring secular terms to vanish gives the solvability conditions:

$$
\begin{aligned}
& 2 i \omega_{m}\left(A^{\prime}+\mu_{m} A\right)+3 \kappa_{m} A^{2} \bar{A}+\frac{1}{2} \alpha_{m} p_{2} \bar{A} e^{i \sigma_{2} T_{1}}=0, \\
& 2 i \tilde{\omega}_{n}\left(\tilde{A}^{\prime}+\tilde{\mu}_{n} \tilde{A}\right)+3 \tilde{\kappa}_{n} \tilde{A}^{2} \tilde{\bar{A}}=0 \\
& 2 i \omega_{\theta}\left(V^{\prime}+\mu_{\theta} V\right)=0
\end{aligned}
$$


where a prime denotes differentiation with respect to $T_{1}$. Letting

$$
\begin{aligned}
A & =\frac{1}{2} a e^{i \varphi}, \quad a, \varphi \in \mathbb{R}, \\
\tilde{A} & =\frac{1}{2} \tilde{a} e^{i \tilde{\varphi}}, \quad \tilde{a}, \tilde{\varphi} \in \mathbb{R}, \\
V & =\frac{1}{2} v e^{i \rho}, \quad v, \rho \in \mathbb{R},
\end{aligned}
$$

and substituting first into (94) gives, when separating real and imaginary parts:

$$
\begin{aligned}
& a^{\prime}=-\mu_{m} a-\frac{1}{4 \omega_{m}} \alpha_{m} p_{2} a \sin \psi_{2} \\
& \psi_{2}^{\prime}=\sigma_{2}-\frac{3}{4 \omega_{m}} \kappa_{m} a^{3}+\frac{1}{2 \omega_{m}} \alpha_{m} p_{2} \cos \psi_{2},
\end{aligned}
$$

where

$$
\psi_{2}=\sigma_{2} T_{1}-2 \varphi
$$

Substituting (102), (97) and (93) into (72), then gives

$$
\xi_{m}=a \cos \left(\frac{1}{2} \Omega_{0} T_{0}+\frac{1}{2} \psi_{2}\right)+O(\varepsilon)
$$

from which it is seen, that in seeking a stationary response of $\xi_{m}$ where the amplitude and phase does not change, we must require $a^{\prime}=\psi_{2}^{\prime}=0$. In this case (100)-(101) has a trivial solution

$$
a=0, \quad \cos \left(\psi_{2}\right)=\frac{2 \sigma_{2} \omega_{m}}{\alpha_{m} p_{2}},
$$

that is, a solution exists for which the span does not vibrate. A non-trivial solution also exists:

$$
a^{2}=\frac{4 \omega_{m}}{3 \kappa_{m}}\left(\sigma_{2} \pm \sqrt{\left(\frac{\alpha_{m} p_{2}}{2 \omega_{m}}\right)^{2}-4 \mu_{m}^{2}}\right), \quad \tan \psi_{2}=\frac{2 \mu_{m}}{\frac{4 \omega_{m} a^{2}}{3 \kappa_{m}}-\sigma_{2}},
$$

when the following conditions are fulfilled,

$$
\left(\frac{\alpha_{m} p_{2}}{2 \omega_{m}}\right)^{2} \geq 4 \mu_{m}^{2} \text { and } \sigma_{2} \pm \sqrt{\left(\frac{\alpha_{m} p_{2}}{2 \omega_{m}}\right)^{2}-4 \mu_{m}^{2}} \geq 0 .
$$


For the solution of $\tilde{A}$ we substitute (98) into (95) and readily solve to find:

$$
\begin{aligned}
& \tilde{a}=\tilde{a}_{0} e^{-\tilde{\mu}_{n} T_{1}}, \\
& \tilde{\varphi}=\tilde{\varphi}_{0}-\frac{3 \tilde{\kappa}_{n} \tilde{a}_{0}^{2}}{\tilde{\mu}_{n} T_{1}} e^{-2 \tilde{\mu}_{n} T_{1}},
\end{aligned}
$$

where $\tilde{a}_{0}$ and $\tilde{\varphi}_{0}$ are arbitrary real-valued constants. Equation (107) shows that $\tilde{a} \rightarrow 0$ as $T_{1} \rightarrow \infty$, since $\tilde{\mu}_{n}>0$, and consequentially $\tilde{\xi}_{n}$ remain small under the specified loading conditions. For the driven sprocket (96) gives the solution

$$
\begin{aligned}
& v=v_{0} e^{-\mu_{\theta} T_{1}}, \\
& \rho=\rho_{0},
\end{aligned}
$$

where $v_{0}$ and $\rho_{0}$ are arbitrary real-valued constants. Here equation (109) shows that $v \rightarrow 0$ as $T_{1} \rightarrow \infty$, since $\mu_{\theta}>0$. Therefore also the driven sprocket angular displacements $u_{1}$ remain small under the specified loading conditions.

Inserting (102) and (93) in (97)-(99) and substituting according to (93) in the oscillatory terms of (88)-(89) the solutions to (81)-(83) can be expressed as

$$
\begin{gathered}
\xi_{m}=a \cos \left(\frac{1}{2} \Omega_{0} T_{0}+\frac{1}{2} \psi_{2}\right)+\varepsilon\left[\frac{k_{m}}{\omega_{m}^{2}}+\frac{\alpha_{m} p_{2} a}{16 \omega_{m}^{2}} \cos \left(\frac{3}{2} \Omega_{0} T_{0}-\frac{1}{2} \psi_{2}\right)\right. \\
+\frac{\alpha_{m} p_{0} a}{\Omega_{0}\left(2 \omega_{m}-\Omega_{0}\right)} \cos \left(\left(\Omega_{0}+\frac{1}{2} \Omega_{2}\right) T_{0}-\frac{1}{2} \psi_{2}\right) \\
-\frac{\alpha_{m} p_{0} a}{\Omega_{0}\left(2 \omega_{m}+\Omega_{0}\right)} \cos \left(\left(\Omega_{0}-\frac{1}{2} \Omega_{2}\right) T_{0}+\frac{1}{2} \psi_{2}\right) \\
+\frac{\kappa_{m} a^{3}}{4 \omega_{m}^{2}} \cos \left(\frac{3}{2}\left(\Omega_{2} T_{0}-\psi_{2}\right)\right) \\
\left.+\sum_{j=1}^{\infty} \frac{2 k_{m}}{\omega_{m}^{2}-\left(j \Omega_{0}\right)^{2}} \cos \left(j \Omega_{0} T_{0}\right)\right]+O\left(\varepsilon^{2}\right)
\end{gathered}
$$




$$
\begin{gathered}
\tilde{\xi}_{n}=\varepsilon\left[\frac{\tilde{k_{n}}}{\tilde{\omega}_{n}^{2}}+\sum_{j=1}^{\infty} \frac{2 \tilde{k}_{n}}{\tilde{\omega}_{n}^{2}-\left(j \Omega_{0}\right)^{2}} \cos \left(j \Omega_{0} T_{0}+\phi\right)\right]+O\left(\varepsilon^{2}\right), \\
u_{1}=\varepsilon\left[\frac{2 \eta_{m} a^{2}}{\omega_{\theta}^{2}}+\frac{2 \eta_{m} a^{2}}{\omega_{\theta}^{2}-\Omega_{2}^{2}} \cos \left(\Omega_{2} T_{0}-\psi_{2}\right)\right. \\
\left.+\frac{\omega_{\theta}^{2} p_{2}}{\omega_{\theta}^{2}-\Omega_{2}^{2}} \cos \left(\Omega_{0} T_{0}\right)+\frac{p_{1}}{\omega_{\theta}^{2}-\Omega_{1}^{2}} \cos \left(\Omega_{1} T_{0}\right)\right]+O\left(\varepsilon^{2}\right) .
\end{gathered}
$$

By assuming the external excitation of the driven sprocket coming from $p_{2}$ to be non-resonant and of order $O(\varepsilon)$, as prescribed by the term $\varepsilon \omega_{\theta}^{2} u_{2}$ in (70), we in effect render the driven sprocket with a zero vibratory response, i.e. the driven sprocket is not compliant to the driver sprocket excitation. For this to hold in the case where the external excitation of the driven sprocket coming from $p_{2}$ is not weak, but $O(1)$, the frequencies $\Omega_{2}$ and $\omega_{\theta}$ should be so far apart that the driven sprocket forced response given by $\omega_{\theta}^{2} p_{2} /\left(\omega_{\theta}^{2}-\Omega_{2}^{2}\right) \cos \left(\Omega_{2} T_{0}\right)$ becomes $O(\varepsilon)$. With this we have demonstrated under which conditions span vibrations decouple from the remaining system, with the response being similar to the decoupled motion of an axially moving string.

\subsection{Primary external resonance of driven sprocket}

During normal operation of a real chain drive, the nominal (angular) velocity varies according to the desired operating conditions. As the excitation from the driver is often period with the driver nominal angular velocity, it is relevant to examine the case where the external excitation is near-resonant to the driven sprocket, $\Omega_{2} \approx \omega_{\theta}$. The other direct and parametric excitations are assumed non-resonant. To describe the nearness of external resonance, we introduce the detuning parameter $\sigma_{\theta}$ by

$$
\omega_{\theta}=\Omega_{2}+\varepsilon \sigma_{\theta}
$$


Inserting into (87)-(89) and requiring secular to terms vanish we obtain the solvability conditions,

$$
\begin{aligned}
& 2 i \omega_{m}\left(A^{\prime}+\mu_{m} A\right)+3 \kappa_{m} A^{2} \bar{A}=0, \\
& 2 i \tilde{\omega}_{n}\left(\tilde{A}^{\prime}+\tilde{\mu}_{n} \tilde{A}\right)+3 \tilde{\kappa}_{n} \tilde{A}^{2} \tilde{\bar{A}}=0, \\
& 2 i \omega_{\theta}\left(V^{\prime}+\mu_{\theta} V\right)-\frac{1}{2} \omega_{\theta}^{2} p_{2} e^{-i \sigma_{\theta}}=0,
\end{aligned}
$$

which are decoupled. Equations (115) and (116) have the same form as (95), thus the response for $\xi_{m}$ and $\tilde{\xi}_{n}$ will decay to zero in the stationary state. Inserting (99) into (117), and introducing

$$
\psi_{\theta}=\sigma_{\theta} T_{1}+\rho
$$

the modulation equations becomes

$$
\begin{aligned}
v^{\prime} & =-\mu_{\theta} v-\frac{1}{2} \omega_{\theta} p_{2} \sin \psi_{\theta}, \\
v \psi_{\theta}^{\prime} & =v \sigma_{\theta}-\frac{1}{2} \omega_{\theta} p_{2} \cos \psi_{\theta} .
\end{aligned}
$$

Back substitution as in the previous case gives $\nu_{0}=v \cos \left(\Omega_{2} T_{0}+\psi_{\theta}\right)$, from which we require $v^{\prime}=\psi_{\theta}^{\prime}=0$ for stationary solutions. Using this in (119)(120) we note that $v=0$ is not a solution, and the stationary amplitude and phase are given by

$$
v=\frac{1}{2} \frac{\omega_{\theta} p_{2}}{\sqrt{\mu_{\theta}^{2}+\left(\omega_{\theta}-\Omega_{2}\right)^{2}}}, \quad \tan \psi_{\theta}=\frac{-\mu_{\theta}}{\omega_{\theta}-\Omega_{2}} .
$$


With $A=\tilde{A}=0$ and $V$ given by (99) with (121)-(121) and (118), the solution of (87)-(89) and (81)-(83) becomes

$$
\begin{aligned}
\xi_{m} & =\varepsilon\left[\frac{k_{m}}{\omega_{m}^{2}}+\sum_{j=1}^{\infty} \frac{2 k_{m}}{\omega_{m}^{2}-\left(j \Omega_{0}\right)^{2}} \cos \left(j \Omega_{o} T_{0}\right)+O\left(\varepsilon^{2}\right)\right], \\
\tilde{\xi}_{n} & =\varepsilon\left[\frac{\tilde{k_{n}}}{\tilde{\omega}_{n}^{2}}+\sum_{j=1}^{\infty} \frac{2 \tilde{k}_{n}}{\tilde{\omega}_{n}^{2}-\left(j \Omega_{0}\right)^{2}} \cos \left(j \Omega_{0} T_{0}+\phi\right)+O\left(\varepsilon^{2}\right)\right], \\
u_{1} & =v \cos \left(\Omega_{2} T_{0}+\psi_{\theta}\right)+\varepsilon\left[\frac{p_{1}}{\omega_{\theta}^{2}-\Omega_{1}^{2}} \cos \left(\Omega_{1} T_{0}\right)\right]+O\left(\varepsilon^{2}\right) .
\end{aligned}
$$

This case demonstrates that for-near resonant excitation of the driven sprocket there will be no transverse vibrations of the spans, if the excitation frequency is not near primary parametric resonance of the spans. However, this is under the assumption that the parametric excitation of the chain spans is small compared to the linear axial stiffness of the chain spans, as assumed in (68)-(69), which might not be fulfilled under large resonant vibrations of the driven sprocket. However, in real chain drives, the ratio between linear axial stiffness and parametric excitation can be decreased by increasing the pretension $P_{t o t}$. We note from (121) that, with the present model, rotational damping of the driven sprocket is the only mechanism which will limit the response of the driven sprocket subjected to resonant excitation. For a real roller chain drive this case indicates that for a chain drive in operation, passing a natural frequency of the driven sprocket does not lead to transverse span vibrations if the excitation is not near parametric resonance of the spans, and that rotational damping of the driven sprocket may reduce the resonant response.

\subsection{Combined parametric and internal resonance between span and sprocket}

In the first example (section 5.10) we analyzed the span response when the driven sprocket is not compliant, i.e. the driver excitation let to a 
vibratory response of the chain span only. The second example (section 5.11) demonstrated the response in the case where the driver excitation caused a vibratory response of the driven sprocket only. Here we analyze the case where the driver excitation may cause a vibratory response of both the driven sprocket and the chain span. This is both of practical interest, and novel to the study of the dynamics of axially moving strings.

Thus we consider primary parametric excitation of the tight span $\Omega_{2} \approx$ $2 \omega_{m}$ combined with internal two-to-one resonance between span and the driven sprocket $\omega_{\theta} \approx 2 \omega_{m}$. All remaining excitations are assumed nonresonant, and the two spans are detuned by loading of the driven sprocket, such that $\omega_{m}$ is away from $\tilde{\omega}_{n}$. To describe the nearness to primary parametric resonance we introduce the detuning parameter $\sigma_{2}$ defined by (93). Similarly, to describe the nearness to internal resonance we introduce the detuning $\sigma_{i}$ defined by

$$
\omega_{\theta}=2 \omega_{m}+\varepsilon \sigma_{i}
$$

Inserting into (87)-(89) and equating to zero the resulting secular terms gives the solvability conditions,

$$
\begin{aligned}
& 2 i \omega_{m}\left(A^{\prime}+\mu_{m} A\right)+3 \kappa_{m} A^{2} \bar{A}-\alpha_{m} \bar{A} V e^{i \sigma_{i} T_{1}}+\frac{1}{2} \alpha_{m} p_{2} \bar{A} e^{i \sigma_{2} T_{1}}=0, \\
& 2 i \tilde{\omega}_{n}\left(\tilde{A}^{\prime}+\tilde{\mu}_{n} \tilde{A}\right)+3 \tilde{\kappa}_{n} \tilde{A}^{2} \tilde{\bar{A}}=0, \\
& 2 i \omega_{\theta}\left(V^{\prime}+\mu_{\theta} V\right)-\eta_{m} A^{2} e^{-i \sigma_{i} T_{1}}-\frac{1}{2} \omega_{\theta}^{2} p_{2} e^{i\left(\sigma_{2}-\sigma_{i}\right) T_{1}}=0 .
\end{aligned}
$$

The condition for $\tilde{A}$ is decoupled, and when represented in polar form (95) the solution is given by (107)-(108), showing that $\tilde{A} \rightarrow 0$ as $T_{1} \rightarrow \infty$. The two other conditions are coupled. Using (97) and (99) the modulation 
equations (126) and (128) becomes:

$$
\begin{aligned}
a^{\prime} & =-\mu_{m} a-\Lambda_{1} a v \sin \psi_{1}-f_{1} a \sin \psi_{2}, \\
v^{\prime} & =-\mu_{\theta} v+\Lambda_{2} a^{2} \sin \psi_{1}-f_{2} \sin \left(\psi_{2}-\psi_{1}\right), \\
a \varphi^{\prime} & =\Lambda_{0} a^{3}+\Lambda_{1} a v \cos \psi_{1}+f_{1} a \cos \psi_{2}, \\
v \rho^{\prime} & =\Lambda_{2} a^{2} \cos \psi_{1}+f_{2} \cos \left(\psi_{2}-\psi_{1}\right),
\end{aligned}
$$

where

$$
\begin{aligned}
\psi_{1} & =\sigma_{i} T_{1}-2 \varphi+\rho, \\
\psi_{2} & =\sigma_{2} T_{1}-2 \varphi \\
\Lambda_{0}=\frac{3 \kappa_{m}}{8 \omega_{m}}, \Lambda_{1}=\frac{-\alpha_{m}}{4 \omega_{m}}, \Lambda_{2} & =\frac{-\eta_{m}}{4 \omega_{\theta}}, f_{1}=\frac{\alpha_{m} p_{2}}{4 \omega_{m}}, f_{2}=-\frac{1}{2} \omega_{\theta} p_{2} .
\end{aligned}
$$

Back substitution gives $q_{0}=a \cos \left(\frac{1}{2} \Omega_{2} T_{0}-\frac{1}{2} \psi_{2}\right)$ and $\nu_{0}=v \cos \left(\Omega_{2} T_{0}+\psi_{2}-\right.$ $\left.\psi_{1}\right)$. Thus, for stationary solutions of (129)-(132) we require $a^{\prime}=v^{\prime}=\psi_{1}^{\prime}=$ $\psi_{2}^{\prime}=0$, which with (133)-(134) gives the equations governing stationary amplitudes:

$$
\begin{aligned}
0 & =-\mu_{m} a-\Lambda_{1} a v \sin \psi_{1}-f_{1} a \sin \psi_{2}, \\
0 & =-\mu_{\theta} v+\Lambda_{2} a^{2} \sin \psi_{1}-f_{2} \sin \left(\psi_{2}-\psi_{1}\right), \\
\frac{1}{2} a \sigma_{2} & =\Lambda_{0} a^{3}+\Lambda_{1} a v \cos \psi_{1}+f_{1} a \cos \psi_{2}, \\
v\left(\sigma_{2}-\sigma_{i}\right) & =\Lambda_{2} a^{2} \cos \psi_{1}+f_{2} \cos \left(\psi_{2}-\psi_{1}\right) .
\end{aligned}
$$

There is a trivial solution given by,

$$
a=0, \quad v^{2}=\frac{f_{2}^{2}}{\mu_{\theta}^{2}+\left(\sigma_{2}-\sigma_{i}\right)^{2}}, \quad \tan \left(\psi_{2}-\psi_{1}\right)=\frac{\mu_{\theta}}{\sigma_{i}-\sigma_{2}},
$$

Representing pure rotational motion of the driven sprocket without transverse vibrations of the upper span, as examined in section 5.11. As for 
non-trivial solutions $a \neq 0$, corresponding to coupled motions of the driven sprocket and the tight span, the algebraic set of nonlinear equations (136)(139) are not readily solved for $a$ and $v$; here we must rely on numerical solution.

\section{Example results of the dynamic analysis}

To illustrate results of the above three resonance cases, we present solutions for a specific chain drive, using configuration $C_{1}$ in Table 1 with pitch $p=0.0254 \mathrm{~m}$, and mass, stiffness, damping and loading parameter values as listed in Table 2 . We consider the first mode $m=1$. With a stationary an-

Table 2: Dynamic parameters

\begin{tabular}{rrl}
\hline$P_{\text {tot }}$ & 1000 & $\mathrm{~N}$ \\
$E A$ & $0.56 \mathrm{e} 6$ & $\mathrm{~N}$ \\
$\rho A$ & 2.61 & $\mathrm{~kg} / \mathrm{m}$ \\
$\hat{J}_{1}$ & 0.6 & $\mathrm{~kg} \mathrm{~m}{ }^{2}$ \\
$\hat{f}_{1}$ & 200 & $\mathrm{~N}$ \\
$\hat{d}_{1}$ & 1.1 & $\mathrm{Nms} / \mathrm{rad}$ \\
$\theta_{2}^{*}$ & 0.005 & $\mathrm{rad}$ \\
$\mu_{\theta}$ & 0.02 & \\
$\mu_{m}$ & 0.02 & \\
\hline
\end{tabular}

gular velocity of the driver of $400 \mathrm{rpm}$ there will then be internal resonance between the tight span and the driven sprocket.

\subsection{Parametric resonance of the tight span}

Here we present an example of the results derived in section 5.10 for the response of the tight span under primary parametric excitation. The 
analysis demonstrated that the tight span transverse motion decouples, because the driven sprocket is non-compliant. The solution for the steady state span vibration amplitude $a$ given by (105) is shown in Figure 6 along with numerical solutions of the modulation equations (100)-(101) shown with circles $\circ$. Full and broken lines identifies stable and unstable solution states, respectively.

The resonance peak near $\Omega_{2} / \omega_{m}=2$ bends to the right due to nonlinear hardening coming from increased axial tension at large amplitudes, represented by the cubic nonlinearity in the model. Increased parametric excitation amplitude $p_{2}$ and decreased damping $\mu_{m}$ widens the resonance peak. It is seen that as the upper branch bends to the right, there are two co-existing stable solutions; one with zero amplitude and another with a large amplitude. Since this is a non-linear response, the solution to which the system converges depends on the initial conditions, which for the numerical solutions were chosen at random, to demonstrate the presence of coexisting solution branches.

In real chain drives, adjusting the span tension to change the natural frequency of the span can be used as an approach to ensure that the the span does not operate near the critical excitation frequencies.

\subsection{Resonant excitation of driven sprocket}

The solution for the driven sprocket steady state amplitude $v$ given by (121) is shown in Figure 7 for the resonant excitation of the driven sprocket, as analyzed in section 5.11. Near $\Omega_{2} / \omega_{\theta}=1$ there is a clearly defined maximum amplitude and the analytical result in (121) shows that increased damping reduces the height of the resonance peak.

In this example the sprocket motion decouples since the spans are ten- 


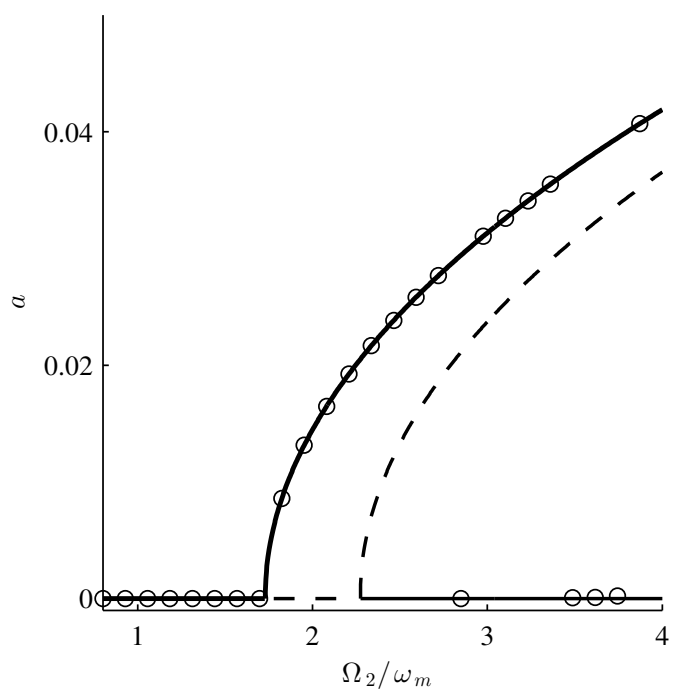

Figure 6: The tight span transverse vibration amplitude $a$ at primary parametric resonance according to perturbation results (105) (solid lines stable, dashed line unstable), and numerical solution of the modulation equations (100)-(101) (circles).

sioned to prevent transverse vibration. Therefore, the response is essentially linear, and the numerical solution confirms there is only a single, independent of initial conditions. The analysis presupposes that the parametric excitation amplitude of the spans is small compared to the span linear stiffness, and the solution is only a good approximation when these conditions are fulfilled; they could be violated during large resonant vibration amplitudes of the driven sprocket, which should therefore still be avoided in real roller chain drives.

\subsection{Combined parametric resonance and internal resonance}

The combination of internal resonance and parametric resonance was investigated in section 5.12. The solution for the span and sprocket steady state response is obtained through numerical integration of the modulation 


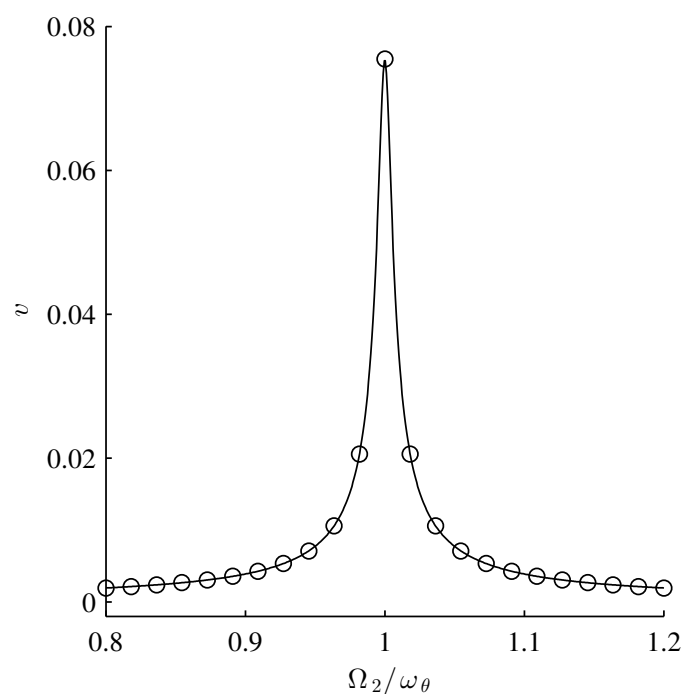

Figure 7: The driven sprocket angular vibration amplitude $v$ at resonant direct external excitation according to the perturbation results (121) (solid line), and numerical solutions of the modulation equations solutions (119)-(120) (circles).

equations (129)-(132). Results for the stationary amplitudes $a$ of the span and $v$ of the driven sprocket are shown in Figures 8 and 9, respectively. In these figures exact parametric resonance occurs for $\sigma_{2}=0$, and exact internal resonance occurs on the line running diagonally across the graphs where $\sigma_{i}=\sigma_{2}$. Figure 8(a) shows three projections of the span vibration amplitude for three values of the detuning of internal resonance $\sigma_{i}$, as indicated by bold lines in Figure 8(b). First we point to the qualitative and quantitative similarity between the bended resonance peak in the response of the decoupled span motion in Figure 6 and the response drawn with solid bold lines for $\sigma_{i} / \omega_{m}=-1.9$ in Figure 8(a) and at the edge of Figure 8(b). For $\sigma_{i} / \omega_{m}=-1.9$ the amplitude $a$ is zero until $\sigma_{2} / \omega_{m}>-0.3$. For $\sigma_{2} / \omega_{m}>0.5$ the stationary amplitude jumps between two solution states. This is similar to what is shown in Figure 6, where the span motion was decoupled, and 

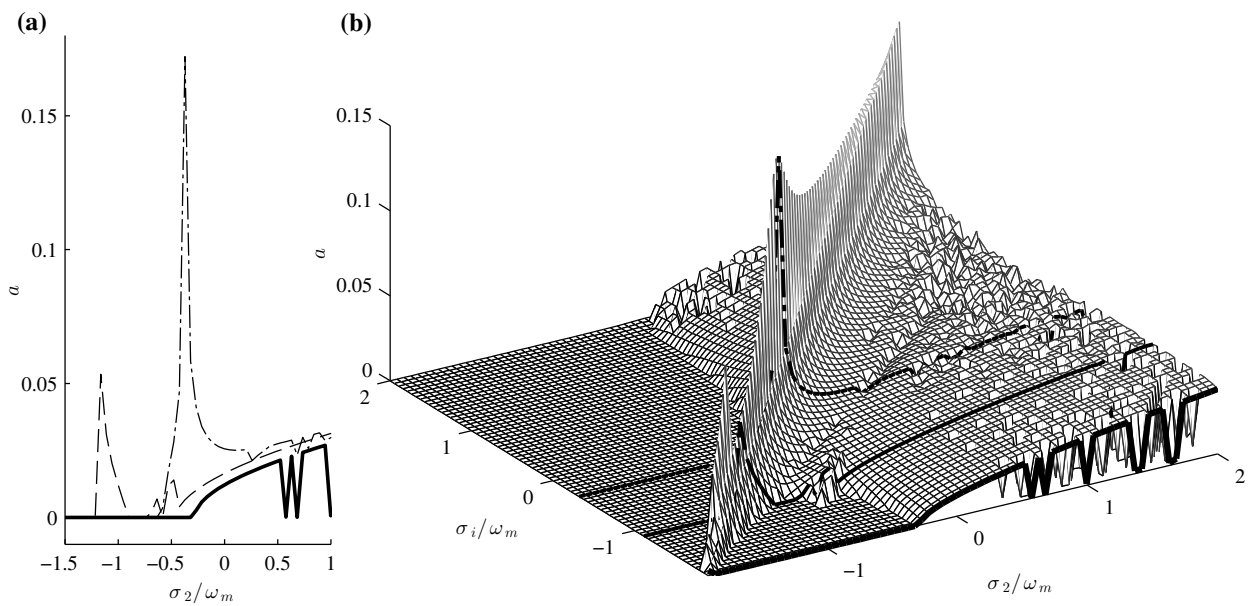

Figure 8: Span transverse vibration $a$ under combined primary parametric resonance and internal resonance obtained by integration of the modulation equations (129)-(132). In (a) cross sections of (b) for values $\sigma_{i} / \omega_{m} \simeq[-1.9,-1.1,-0.3]$ are shown with lines $[-,--,-\cdot-]$, respectively.

it also appears from Figure 8 that for $\sigma_{2} / \omega_{m}>0.5$ there are two coexisting solutions between which the response jumps between depending on the initial conditions.

Secondly, we observe in Figure 8 that there is a large upright amplitude peak running diagonally across Figure 8(b) near exact combined parametric and internal resonance $\sigma_{i}=\sigma_{2}$. As $\sigma_{i} / \omega_{m}=\sigma_{2} / \omega_{m}$ increase from below to approach zero, the upright resonant peak combines with the bended resonance peak and the amplitude reaches maximum for $\sigma_{i} / \omega_{m}=$ $\sigma_{2} / \omega_{m} \simeq-0.3$. The upright peak is present for values of $\sigma_{2} / \omega_{m}$ much lower than zero, and the upright and bended resonance peaks are separated for $\sigma_{i} / \omega_{m}<-1.1$. This indicate that when the driven sprocket is compliant, chain span vibrations excited by parametric excitation can occur for much lower values of $\sigma_{2} / \omega_{m}$ than when the span is decoupled, which is a significant 
and novel result. In real chain drives, this means that span transverse vibration can be excited at frequencies lower than parametric resonance when the driven sprocket has a vibratory response.
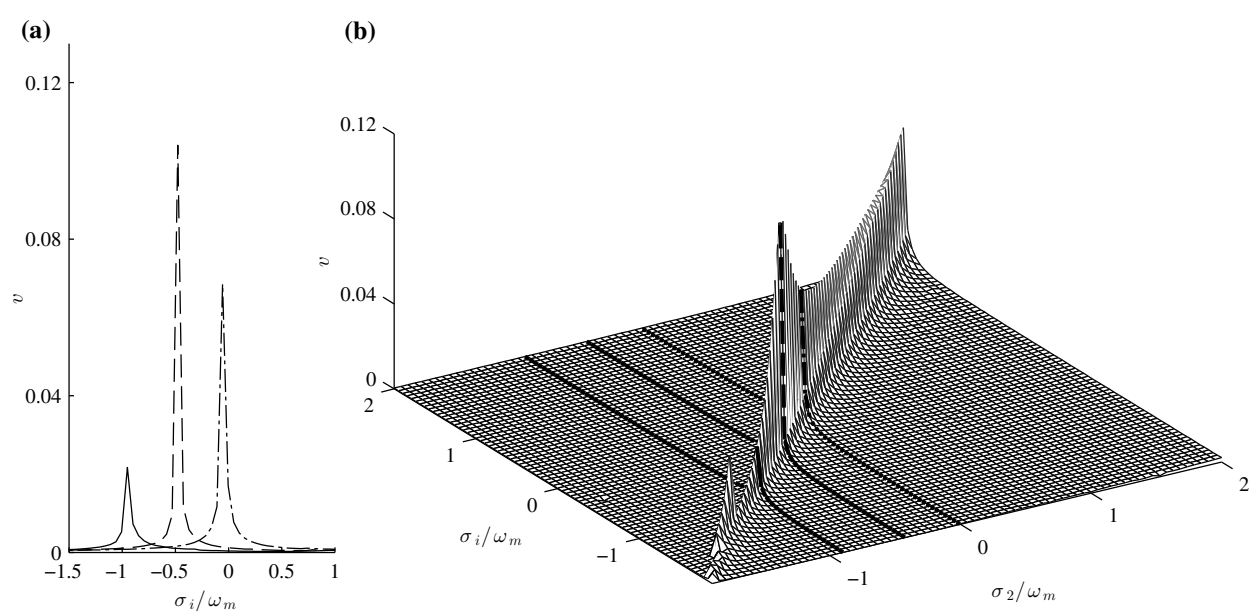

Figure 9: Driven sprocket angular vibration $v$ under combined parametric and internal resonance obtained by integration of the modulation equations (129)-(132). In (a) cross sections of (b) for values $\sigma_{2} / \omega_{m} \simeq[-0.9,-0.5,0]$ are shown with lines $[-,--,-\cdot-]$, respectively.

Figure 9(a) shows three projections of the driven sprocket amplitude for three values of the detuning of parametric resonance $\sigma_{2}$ indicated with bold lines in Figure 9(b). We observe a qualitative and partly quantitative similarity to the upright resonance peak in the response of driven sprocket motion in Figure 7 and the cross sections in Figure 9(a). The maximum value of $v$ is observed for $\sigma_{2} / \omega_{m} \simeq-0.5$.

In both Figure 8(b) and 9(b) we observe an amplitude peak around $\sigma_{i} / \omega_{m}=\sigma_{2} / \omega_{m} \simeq-1.5$. The explanation for this may require further analysis. For all the presented examples we note that the response is obtained using a perturbation method assuming near-resonance. Thus, predictions 
will be most accurate near $\sigma_{i}=\sigma_{2}=0$.

\section{Conclusion(s)}

1. A kinematic model of a two-sprocket roller chain drive with straight spans was presented, along with a procedure for calculating the total chain wrapping length. Analytical predictions were compared to multibody simulation results and demonstrated that the total wrapping length of the chain generally varies periodically with the tooth frequency. The chain wrapping length was shown to be constant for a configuration where both sprockets have the same number of teeth and the chain consists of an even number of chain links.

2. Since the chain wrapping length generally varies during rotation of the sprockets, it is hypothesized (and left for future studies) that careful positioning of more than two sprockets can be done so as to attenuate or amplify the effect of a variable wrapping length.

3. Deriving the equations of motion for the chain spans we treated the problem of an axially moving string supported by moving boundaries, and showed that a first order variable string length leads to a second order effect.

4. We presented a new dynamic model for the coupled motion of the tight chain spans transverse vibration and the driven sprocket angular displacement. The model assumed the presence of a steady operation state from which displacements are measured.

5. The dynamic model provides insight into resonance conditions and amplitude responses, and was analyzed approximately using a mode shape expansion and perturbation analysis. 
6. Three example results of the dynamic analysis were presented, illustrating the conditions where the motions of the span and sprockets decouple. Results for a case of combined internal and parametric resonance showed that large span vibrations can occur due to compliance of the driven sprocket.

7. Though the model is simple, it provides useful insight into the coupled dynamics of chain drives, and may aid the design and interpretation of numerical and experimental results.

8. Due to the systematic structure of multiple scales analysis presented here, where the modulation equations decouple for non-resonant excitation, it is expected that the dynamics of chain drives with more than two sprockets could be analyzed using a similar modeling and analysis approach. In the analysis presented here, the coupled motion of transverse span vibrations happens across a single degree of freedom oscillator, which is the driven sprocket with a given natural frequency. For a larger chain drive with more sprockets, this would correspond to modal excitation near the natural frequencies of the coupled rotational motion of the sprockets with the spans acting as linear springs.

\section{References}

[1] K. W. Wang and S. P. Liu. On the noise and vibration of chain drive systems. The Shock and Vibration Digest, 23(4):8-13, 1991.

[2] G. M. Bartlett. Roller chain drives in theory and practice. Product Engineering, 2(4):253-255, 1931.

[3] R. A. Morrison. Polygonal action in chain drives. Machine Design, 24 (9):155-159, 1952. 
[4] Raymond Charles Binder. Mechanics of the Roller Chain Drive: Based on Mathematical Studies by RC Binder. Prentice-Hall, 1956.

[5] G. Bouillon and G. V. Tordion. On polygonal action in roller chain drives. Journal of Engineering for Industry, 87(2):243-250, 1965.

[6] C. K. Chen and F. Freudenstein. Toward a more exact kinematics of roller chain drives. Journal of Mechanisms Transmissions and Automation in Design, 110(3):269-275, 1988.

[7] N. Fuglede and J. J. Thomsen. Kinematics of roller chain drives - exact and approximate analysis. Submitted for Journal Publication, 2014.

[8] S. L. Pedersen, J. M. Hansen, and J. A. C. Ambrosio. A roller chain drive model including contact with guide-bars. Multibody System Dynamics, 12(3):285-301, 2004.

[9] S. L. Pedersen. Model of contact between rollers and sprockets in chaindrive systems. Archive of Applied Mechanics, 74(7):489-508, 2005.

[10] A. L. Thurman and C. D. Mote, Jr. Free periodic nonlinear oscillation of an axially moving strip. Journal of Applied Mechanics, 36(1):83-91, 1969.

[11] C. D. Mote, Jr. and A. L. Thurman. Oscillation modes of an axially moving material. Journal of Applied Mechanics, 38(1):279-280, 1971.

[12] L. Meirovitch. A new method of solution of the eigenvalue problem for gyroscopic systems. AIAA Journal, 12(10):1337-1342, 1974.

[13] L. Meirovitch. Modal analysis for response of linear gyroscopic systems. Journal of Applied Mechanics, 42(2):446-450, 1975. 
[14] J. A. Wickert and C. D. Mote, Jr. Classical vibration analysis of axially moving continua. Journal of Applied Mechanics, 57(3):738-744, 1990.

[15] J. A. Wickert. Nonlinear vibration of a traveling tensioned beam. International Journal of Non-Linear Mechanics, 27(3):503-517, 1992.

[16] E. M. Mockensturm, N. C. Perkins, and A. G. Ulsoy. Stability and limit cycles of parametrically excited, axially moving strings. Journal of Vibration and Acoustics, 118(3):346-351, 1996.

[17] Li-Qun Chen. Analysis and control of transverse vibrations of axially moving strings. Applied Mechanics Review, 58(2):91-116, 2005. ISSN 00036900,10888535 .

[18] R. G. Parker and Y. Lin. Parametric instability of axially moving media subjected to multifrequency tension and speed fluctuations. Journal of Applied Mechanics, 68(1):49-57, 2001.

[19] J. A. Wickert and C. D. Mote, Jr. Current research on the vibration and stability of axially-moving materials. The Shock and Vibration Digest, 20(5):3-13, 1988.

[20] S. Mahalingam. Transverse vibrations of power transmission chains. British Journal of Applied Physics, 8(4):145-148, 1957.

[21] S. Mahalingam. Polygonal action in chain drives. Journal of The Franklin Institute, 265(1):23-28, 1958. ISSN 00160032.

[22] S. T. Ariaratnam and S. F. Asokanthan. Dynamic stability of chain drives. Journal of Mechanisms Transmissions and Automation in Design, 109(3):412-418, 1987. 
[23] K. W. Wang and Jr. Mote, C. D. Vibration coupling analysis of band/wheel mechanical systems. Journal of Sound and Vibration, 109 (2):237-258, 1986. ISSN 0022460x, 10958568.

[24] K. W. Wang. On the stability of chain drive systems under periodic sprocket oscillations. Journal of Vibration and Acoustics, 114(1):119126, 1992.

[25] R.C. Binder and W.V. Covert. Impact between chain roller and sprocket in chain drive. Franklin Institute - Journal, 245(4):319-329, 1948.

[26] G. K. Ryabov. Inertia effects of impact loading in chain drives. Russian Engineering Journal, 48(8):17-19, 1968.

[27] J. N. Fawcett and S. W. Nicol. Vibration of a roller chain drive operating at constant speed and load. Proceedings of the Institution of Mechanical Engineers, 194:97-101, 1980.

[28] J. C. Conwell and G. E. Johnson. Experimental investigation of link tension and roller-sprocket impact in roller chain drives. Mechanism and Machine Theory, 31(4):533-544, 1996.

[29] K. W. Wang, S. P. Liu, S. I. Hayek, and F. H. K. Chen. On the impact intensity of vibrating axially moving roller chains. Journal of Vibration and Acoustics, 114(3):397-403, 1992.

[30] G K Ryabov and A V Kryukov. Shock loads in chain transmissions. Russian Engineering Research, 17(6):15, 1997.

[31] S. P. Liu, K. W. Wang, S. I. Hayek, M. W. Trethewey, and F. H. K. Chen. A global-local integrated study of roller chain meshing dynamics. Journal of Sound and Vibration, 203(1):41-62, 1997. 
[32] H. Zheng, Y. Y. Wang, G. R. Liu, K. Y. Lam, K. P. Quek, T. Ito, and Y. Noguchi. Efficient modelling and prediction of meshing noise from chain drives. Journal of Sound and Vibration, 245(1):133-150, 2001.

[33] Mohammad R. Naji and Kurt M. Marshek. Analysis of sprocket load distribution. Mechanism and Machine Theory, 18(5):349-356, 1983.

[34] Mohammad R. Naji and Kurt M. Marshek. Effects of the pitch difference on the load ditribution of a roller chain drive. Mechanism and Machine Theory, 24(5):351-362, 1989.

[35] Mahn Shik Kim and Glen E. Johnson. Mechanics of roller chainsprocket contact: a general modelling stragegy. American Society of Mechanical Engineers, Design Engineering Division (Publication) DE, 43(2):689-695, 1992.

[36] L. Meirovitch. Fundamentals of Vibrations. McGraw-Hill Higher Education, 2001.

[37] Cornelius Lanczos. The variational principles of mechanics. University of Toronto Press, 1966.

[38] J. A. Wickert and C. D. Mote, Jr. On the energetics of axially moving continua. Journal of the Acoustical Society of America, 85(3):1365$1368,1989$.

[39] W. D. Zhu and J Ni. Energetics and stability of translating media with an arbitrarily varying length. Journal of Vibration and Acoustics, 122 (3):295-304, 2000. 


\section{Nomenclature}

\section{Latin}

$a\left(T_{1}\right) \quad$ Real-valued slow modulation amplitude for transverse vibrations

A $\quad$ Cross section area of string

$A_{j}-D_{j} \quad$ Chain drive configurations, $j=1,2,3$ (def. in Table 1 )

$A\left(T_{1}\right) \quad$ Complex-valued slow modulation amplitude for transverse vibrations

$c_{u}, c_{w} \quad$ Wave speed of transverse and axial string waves, resp.

C Driver sprocket

$C_{m n} \quad$ Modal expansion coefficient (def. in Eq. (59))

$d \quad \hat{d}_{1}$ non-dimensionalized by $R_{1}^{2} \sqrt{\rho A P_{t}}$

$\hat{d}_{1} \quad$ Rotational viscous damping coefficient for driven sprocket

$D_{j}^{k}\left(\right.$ ) $\quad k$ 'th partial derivative with respect to $T_{j}$

$e \quad$ Axial strain with mean value subtracted

$e_{0} \quad$ Mean axial strain

E Young's modulus of string material

$E_{m} \quad$ Modal expansion coefficient (def. in Eq. (59))

$f \quad$ Pitch fraction

$f_{2} \quad$ Rotation frequency (in $\mathrm{Hz}$ ) of driver sprocket

$f_{m}(t) \quad m$ 'th modal forcing component

$\hat{f}_{1} \quad$ Brake load

$G_{m} \quad$ Modal expansion coefficient (def. in Eq. (59))

$h \quad$ Binary function (def. in Eq. (2))

$i \quad$ imaginary unit

$J \quad \hat{J}_{1}$ non-dimensionalized by $\rho A l R_{1}^{2}$ 


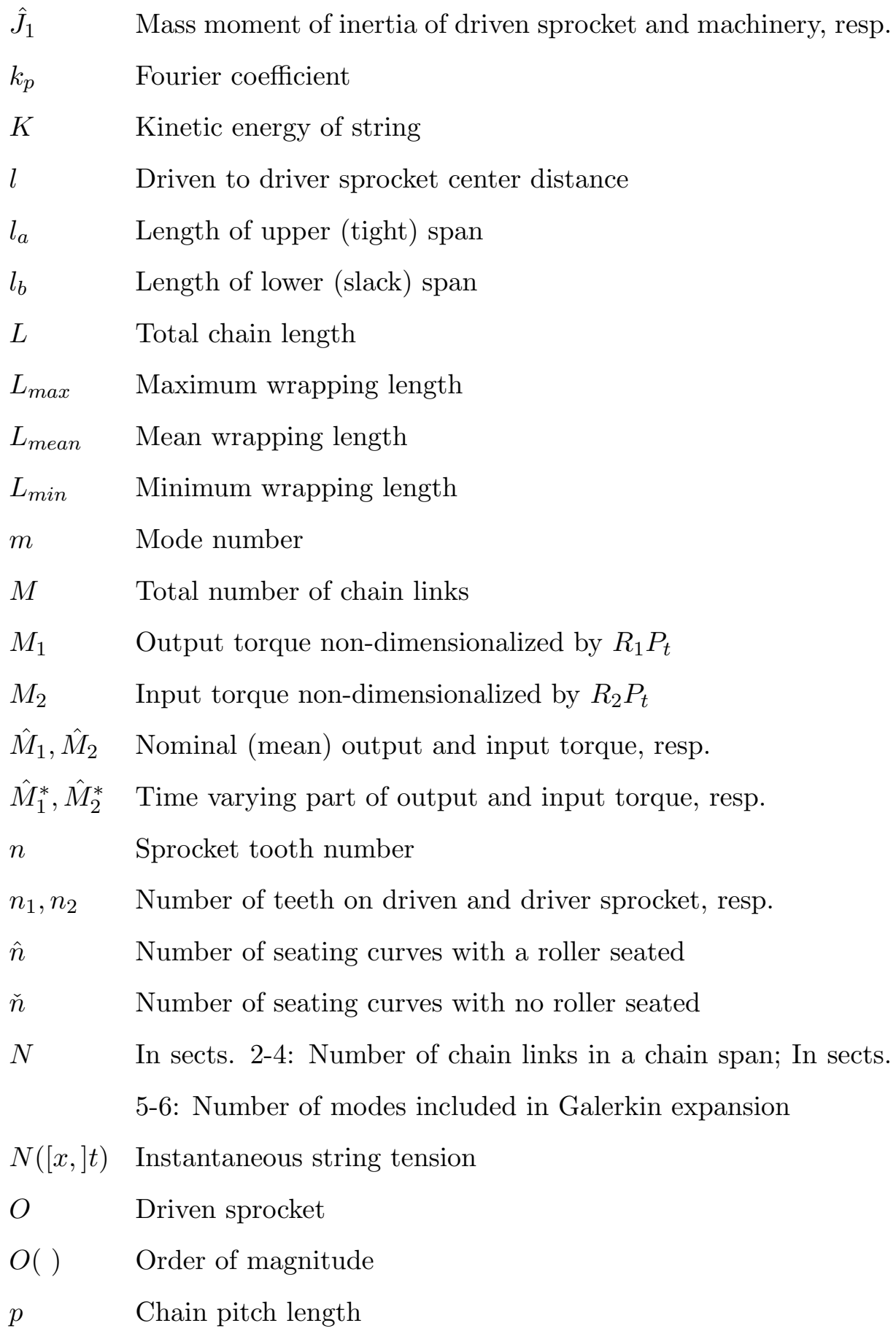


$p_{0}, p_{1}, p_{2}$ Non-dimensional harmonic forcing amplitudes for the singlemode approximation

$p_{0}^{*} \quad$ Additional tension from time-varying wrapping length

$P \quad$ Actual span tension

$P_{0} \quad$ Mean steady state span tension

$P_{\text {pre }} \quad$ Span pretension

$P_{t} \quad$ Reference span tension (for non-dimensionalizing time)

$P_{t o t} \quad$ Total pretension (two spans)

$q_{0}, q_{1} \quad$ Multiple scales expansion functions for $\xi_{m}(t)$

$Q_{1}, Q_{2} \quad$ Acceleration jump at driven and driver sprocket, resp.

$r \quad$ Radius of pitch polygon inscribed circle

$R \quad$ Radius of pitch circle

$R_{1}, R_{2} \quad$ Radius of driven and driver pitch circle, resp.

$s \quad S$ non-dimensionalized by $\sqrt{P_{t} / \rho A}$

$S \quad$ Nominal span velocity

$t \quad$ Time non-dimensionalized by $\sqrt{\rho A l^{2} / P_{t}}$

$t_{u}, t_{l} \quad$ Upper and lower tangent of inscribed sprocket circles

T Time

$T_{0}, T_{1} \quad$ Slow and fast time in Multiple scales analysis

$u \quad U$ non-dimensionalized by $l$

$u_{1}, u_{2} \quad U_{1}, U_{2}$ non-dimensionalized by $l$

$u_{2}^{*} \quad U_{2}^{*}$ non-dimensionalized by $l$

$U \quad$ Axial displacement of chain or string point

$U_{1}, U_{2} \quad$ Axial displacement (wrt. $X_{1}, X_{2}$ ) of moving string endpoints

$U_{2}^{*} \quad$ Prescribed / kinematically forced value of $U_{2}$ 
$v\left(T_{1}\right) \quad$ Real-valued slow modulation amplitude for rotational vibrations of driven sprocket

$V \quad$ Potential (elastic) energy of string

$V\left(T_{1}\right) \quad$ Complex-valued slow modulation amplitude for rotational vibrations of driven sprocket

$w \quad$ Non-dimensionalized transverse chain or string displacement with rigid body mode subtracted

$\hat{w} \quad \hat{W}$ non-dimensionalized by $l$

$\hat{W} \quad$ Transverse displacement of chain or string point

$x, y \quad X, Y$ non-dimensionalized by $l$

$x_{1}, x_{2} \quad X_{1}, X_{2}$ non-dimensionalized by $l$

$X, Y \quad$ Inertial coordinate system for upper chain span

$X_{1}, X_{2} \quad$ Left and right endpoint coordinates along $X$ of moving string

$X^{\prime}, Y^{\prime} \quad$ Inertial coordinate system for lower chain span

$y_{1}, y_{2} \quad Y_{1}, Y_{2}$ non-dimensionalized by $l$

$Y_{1}, Y_{2} \quad$ Transverse displacement (at $X_{1}, X_{2}$ ) of moving string endpoints

\section{Greek}

$\alpha \quad$ In Sect. 22: Pitch angle; Elsewhere: Axial string stiffness EA non-dimensionalized

$\alpha_{1}, \alpha_{2} \quad$ Pitch angle of driven and driver sprocket, resp.

$\alpha_{m} \quad$ Modal expansion coefficient (def. in Eq. (61))

$\beta \quad$ Angle btw. coord. systems $(X, Y)$ and $\left(X^{\prime}, Y^{\prime}\right)$

$\gamma \quad P_{0}$ non-dimensionalized by $E A$

$\delta($ ) Variation

$\Delta x_{1}, \Delta x_{2}$ Small axial variations of string support positions

$\epsilon \quad$ Small quantity (used for order of magnitude ranking) 
$\eta_{m}, \tilde{\eta}_{n} \quad$ Coupling coefficients (def. in Eq. (71))

$\phi \quad$ Phase shift between upper and lower span acceleration jump

$\phi_{a}, \phi_{b} \quad$ Angle to, resp., upper and lower span endpoint roller for driven sprocket

$\phi_{m}(x) \quad m$ 'th transverse mode shape for axially non-moving uniform string

$\varphi\left(T_{1}\right) \quad$ Real-valued slow modulation phase for transverse string vibrations

$\theta_{1}, \theta_{2}^{*} \quad$ Angular position of driven and driver sprocket, resp.

$\theta_{a}, \theta_{b} \quad$ Angle to, resp., upper and lower span endpoint roller for driver sprocket

$\theta_{a r}, \phi_{a r} \quad$ Upper span release configuration

$\theta_{a s}, \phi_{a s} \quad$ Upper span seating configuration

$\theta_{b r}^{\prime}, \phi_{b r}^{\prime} \quad$ Lower span release configuration

$\theta_{b s}^{\prime}, \phi_{b s}^{\prime} \quad$ Lower span seating configuration

$\kappa_{m} \quad$ Modal expansion coefficient (def. in Eq. (61))

$\mu_{m} \quad$ m'th modal damping coefficient

$\mu_{\theta} \quad$ Damping coefficient for rotational vibrations of driven sprocket

$\nu_{0}, \nu_{1} \quad$ Multiple scales expansion functions for $u_{1}(t)$

$\xi_{m}(t) \quad m$ 'th modal amplitude

$\rho A \quad$ String mass per unit length

$\rho\left(T_{1}\right) \quad$ Real-valued slow modulation phase for rotational vibrations of driven sprocket

$\sigma_{2} \quad$ Detuning: nearness of $\Omega_{2}$ to parametric resonance $2 \omega_{m}$

$\sigma_{\theta} \quad$ Detuning: nearness of $\Omega_{2}$ to external resonance $\omega_{\theta}$

$\sigma_{i} \quad$ Detuning: nearness of $\omega_{\theta}$ to internal resonance $2 \omega_{m}$ 


$$
\begin{array}{ll}
\tau_{0} & \text { Non-dimensional tooth period } \\
\psi & \text { Phase shift between acceleration jump at driver and driven } \\
& \text { sprocket } \\
\psi_{1,2, \theta}\left(T_{1}\right) & \text { Slow phase variables for the multiple scale analysis } \\
\omega_{m} & m^{\prime} \text { th linear natural frequency for upper span } \\
\tilde{\omega}_{n} & n^{\prime} \text { th linear natural frequency for lower span } \\
\omega_{\theta} & \text { Linear natural frequency for rotational vibrations of driven } \\
& \text { sprocket } \\
\Omega_{0} & \text { Fundamental frequency of acceleration jumps at sprockets } \\
\Omega_{1} & \text { Oscillation frequency of external load on driven sprocket } \\
\Omega_{2} & \text { Oscillation frequency of driver sprocket }
\end{array}
$$

\section{Subscript}
a Upper (tight) chain span
$b \quad$ Lower (slack) chain span
$c, 2 \quad$ Driver sprocket
$o, 1 \quad$ Driven sprocket
$r \quad$ Release configuration of chain link
$s \quad$ Seat configuration of chain link

\section{Diacritic and other symbols}

()$^{\prime} \quad$ In sects. 2-4: Variable measured in $\left(X^{\prime}, Y^{\prime}\right)$; In Sect. 5: differentiation wrt. $T_{1}$

( ), Partial differentiation wrt. the variable(s) following the comma

() Parameter or variable for lower (slack) span

( ) Complex conjugate 